\title{
Desarrollo del eje reproductivo endocrino en corderos de pelo
}

\section{Development of the endocrine reproductive axis in hair breed ram lambs}

\author{
Luis Javier Montiel-Olguínab, Héctor Raymundo Vera-Ávilaab, Eugenio Villagómez- \\ Amezcuabc, Vicente Castañeda-Rodrígueza , Mario Cárdenas-Leónd, \\ Héctor Jiménez-Severianoab
}

\begin{abstract}
RESUMEN
Los objetivos fueron caracterizar los perfiles de LH y testosterona ( $T$ ) y la respuesta a GnRH en corderos de pelo. Estudio 1: se utilizaron corderos Blackbelly $(n=4)$, Pelibuey $(n=5)$, Blackbelly x Pelibuey $($ Cruzas; $n=8)$. A las 2,4 , $8,12,16,20$ y 24 semanas de edad, se obtuvieron muestras de sangre cada 15 min durante 6 h, para caracterizar los perfiles de LH y T. No hubo diferencia entre genotipos a ninguna edad, para ninguna variable de $L$ L y $T(P>0.05) ;$ el efecto de la edad fue significativo $(P<0.05)$ : la concentración promedio de LH fue menor en la semana $2(P<0.05)$, alcanzó el valor más alto en la 12, y disminuyó en la $16(P<0.05)$. La concentración promedio de $T$ aumentó constantemente entre 2 y 24 semanas $(P<0.05)$. Estudio 2: se utilizaron corderos Blackbelly $(n=4)$, Pelibuey $(n=4)$, Cruzas $(\mathrm{n}=4)$. A las mismas edades, los corderos se desafiaron con $\mathrm{GnRH}\left(3,9,27 \mathrm{ng}^{\circ} \mathrm{kg}^{-1}\right.$ peso) y se obtuvieron muestras de sangre entre -15 y 180 min. En las semanas 8 y 12, la respuesta de LH fue mayor en los corderos Pelibuey, en comparación con Blackbelly. La respuesta de $\mathrm{T}$ tendió a ser diferente entre genotipos $(P<0.10)$, con Blackbelly mostrando la menor respuesta. En conclusión, el desarrollo de los perfiles de LH y T no fue diferente entre genotipos, pero los corderos Pelibuey presentaron mayor capacidad de respuesta a GnRH en las semanas 8 y 12; es necesario determinar si estas diferencias tienen implicaciones sobre la capacidad reproductiva de los animales adultos.
\end{abstract}

PALABRAS CLAVE: Blackbelly, Pelibuey, LH, Testosterona, Desarrollo sexual.

\begin{abstract}
The objectives were to characterize the profiles of LH and testosterone (T) and the response to GnRH administration in hair ram lambs. Study 1: seventeen lambs were used: Blackbelly $(n=4)$, Pelibuey $(n=5)$, Blackbelly $x$ Pelibuey (Crossbred; $n=8$ ). At 2, 4, 8, 12, 16, 20 and 24 wk of age, blood samples were collected every 15 min for $6 \mathrm{~h}$, to characterize LH and T profiles. There was no significant difference between genotypes at any age, for any variable of $\mathrm{LH}$ and $\mathrm{T}$ profiles $(P>0.05)$. The effect of age was significant $(P<0.05)$ : mean LH concentration was lower on wk $2(P<0.05)$, achieved the highest value on wk 12, and decreased on wk $16(P<0.05)$. Mean $T$ concentration increased steadily between wk 2 and $24(P<0.05)$. Study 2: twelve lambs were used: Blackbelly $(n=4)$, Pelibuey $(n=4)$, Crossbred $(n=4)$. At the same ages, lambs were challenged with GnRH $\left(3,9,27\right.$ ng॰ $\left.\mathbf{k g}^{-1} \mathrm{BW}\right)$ and blood samples were obtained between -15 and 180 min from GnRH administration. On wk 8 and 12, LH response to GnRH was greater in Pelibuey, as compared with Blackbelly. Testosterone response tended to be different between genotypes $(P<0.10)$, with Blackbelly showing the smallest response. In conclusion, the sexual endocrine development, as evaluated by the non-stimulated LH and T profiles, was not different among genotypes; however, Pelibuey elicited the greatest responsiveness to GnRH administration at 8 and 12 wk of age. Whether or not such differences have implications on the reproductive capacity of the adult animals deserves additional research.
\end{abstract}

KEY WORDS: Blackbelly, Pelibuey, LH, Testosterone, Sexual development.

Recibido el 20 de abril de 2015. Aceptado el 20 de julio de 2015.

a Centro Nacional de Investigación Disciplinaria en Fisiología y Mejoramiento Animal, INIFAP. Ajuchitlán, Querétaro, México. jimenez.hector@inifap.gob.mx. Correspondencia al sexto autor.

b Posgrado en Ciencias de la Producción y de la Salud Animal, FES Cuautitlán, UNAM. Cuautitlán Izcalli, Estado de México, México.

c Centro Nacional de Investigación Disciplinaria en Microbiología Veterinaria, INIFAP. Cuajimalpa, DF, México.

d Instituto Nacional de Ciencias Médicas y Nutrición Salvador Zubirán. Tlalpan, DF, México. 


\section{INTRODUCCIÓN}

El desarrollo endocrino sexual ha sido estudiado en corderos de razas de lana(1,2), mientras que en razas tropicales de pelo esta información es escasa. A partir de estudios con razas de lana, se han descrito varios aspectos. Se sabe que hay un eje endocrino activo en los corderos recién nacidos(3), que se desarrolla a medida que crecen(1); los cambios anatómicos e histológicos durante el desarrollo prepuberal se asocian a cambios endocrinos(4); la edad a la maduración sexual difiere entre razas, siendo algunas más precoces que otras, y la precocidad se relaciona con la fertilidad y prolificidad de los adultos(5). Barbados Blackbelly (en adelante simplemente Blackbelly) y Pelibuey son razas de pelo importantes (Ovis aries), desarrolladas en latitudes tropicales $y$, por lo tanto, bien adaptadas a estas condiciones. Blackbelly es considerada una de las razas de pelo más prolíficas(6); más prolífica que la Pelibuey $(7,8)$, pero el tamaño de camada en esta última es mayor que en otras razas tropicales(9); por otro lado, hay controversia en cuanto a si Blackbelly es más precoz que Pelibuey(10-13).

En los corderos Blackbelly se observa un aumento temprano de las concentraciones séricas de la hormona luteinizante (LH) a las 6 semanas de edad, y aumento de la testosterona ( $\mathrm{T})$ en las semanas 12 y 21(14); sin embargo, esta información se basó en muestras individuales; debido a la naturaleza pulsátil de la secreción de LH y T, es necesario obtener muestras con mayor frecuencia para caracterizar con precisión los perfiles hormonales y la sensibilidad del eje hipófisis-testículo. Adicionalmente, al parecer no se ha hecho una comparación directa del desarrollo endocrino entre corderos Blackbelly y Pelibuey; por lo tanto, el objetivo del estudio fue caracterizar el desarrollo del eje reproductivo endocrino en corderos Blackbelly, Pelibuey y Blackbelly $\mathrm{x}$ Pelibuey (cruzas). La hipótesis fue que el eje endocrino se desarrollaría más temprano en los

\section{INTRODUCTION}

The sexual endocrine development has been studied in lambs of wool breeds $(1,2)$, whereas in tropical hair sheep this information is scarce. From studies in wool breeds, several aspects have been described. It is known that there is an active endocrine axis in the new born lambs(3), which develops as the ram grows(1); the anatomical and histological changes during the prepubertal development are associated to endocrine changes(4). The age at sexual maturation differs among breeds, some breeds being more precocious than others, and precocity is related to breed fertility and prolificacy in the adult ewe (5). Barbados Blackbelly (hereafter just Blackbelly) and Pelibuey are important hair breeds (Ovis aries) developed in tropical latitudes and, therefore, well adapted to tropical conditions. Blackbelly is considered one of the most prolific hair breeds( 6$)$; previous studies have shown that Blackbelly ewes are more prolific than Pelibuey ewes $(7,8)$, but litter size in Pelibuey is greater than in other breeds raised in the tropics(9). Some controversy still exists as to whether Blackbelly is more precocious than Pelibuey(10-13).

In Blackbelly male lambs, an early increase in serum LH was observed at 6 wk of age, with testosterone ( $\mathrm{T}$ ) increasing at wk 12 and 21(14); however, this information was based on single samples taken just before castration; because of the pulsatile nature of $\mathrm{LH}$ and T secretion, a more frequent blood sampling is necessary to characterize accurately the hormonal profiles and sensitivity of the hypophysis-testis axis. Furthermore, to our knowledge, comparison, in terms of endocrine development, between Blackbelly and Pelibuey males have not been made; therefore, the objective of this study was to characterize the development of the endocrine reproductive axis in Blackbelly, Pelibuey and Blackbelly $x$ Pelibuey ram lambs. We hypothesize that the endocrine axis will develop earlier in Blackbelly, as compared with Pelibuey lambs, with crossbred lambs being intermediate. 
corderos Blackbelly que en los Pelibuey, siendo intermedios los corderos cruzados.

\section{MATERIAL Y MÉTODOS}

Se realizaron dos estudios utilizando corderos Blackbelly, Pelibuey y Cruzas. En el primer estudio, se evaluó el desarrollo de los perfiles de LH y T circulantes por medio de muestreos sanguíneos seriados. En el segundo estudio, se administraron dosis crecientes de la hormona liberadora de gonadotropinas $(\mathrm{GnRH})$ para evaluar el desarrollo de la respuesta, en términos de la secreción de LH y T.

\section{Condiciones generales}

Los estudios se realizaron en el estado de Querétaro, México (200 42' N; $100^{\circ} 01^{\prime}$ O). Los protocolos y procedimientos experimentales fueron aprobados por el Subcomité Institucional para el Cuidado y Uso de Animales de Experimentación (SICUAE), de la Facultad de Medicina Veterinaria y Zootecnia de la Universidad Nacional Autónoma de México, y de acuerdo con la "International Guiding Principles for Biomedical Research Involving Animals" (http://www.cioms.ch/images/stories/ CIOMS/IGP2012.pdf). Los corderos nacieron entre el 2 y 29 de marzo (estudio 1) o entre el 29 de octubre y 14 de noviembre (estudio 2). Antes del destete (70 días de edad), los corderos se alimentaron con una dieta que contenía 16.9 \% PC y 2.74 Mcal EM/kg MS, y las ovejas con una dieta con $13.04 \%$ PC y $2.33 \mathrm{Mcal} \mathrm{EM} / \mathrm{kg}$ MS. Después del destete, a los corderos se les ofreció una dieta con $14.05 \%$ de PC y 2.65 Mcal EM/kg MS, y agua ad libitum. Un día antes de que cada cordero cumpliera 2, $4,8,12,16,20$ y 24 semanas de edad, se registró el peso corporal, se midió la circunferencia escrotal (CE), como se ha descrito previamente(15), y a cada cordero se le colocó un catéter en la vena yugular. En ese momento $y$ al final de los muestreos, los corderos recibieron complejo B $(0.15 \mathrm{~g}$ de tiamina, $0.2 \mathrm{~g}$ de riboflavina, $0.3 \mathrm{~g}$ de nicotinamida, $0.2 \mathrm{~g}$ de piridoxina, $90 \mu \mathrm{g}$ de cianocobalamina y $0.15 \mathrm{~g}$

\section{MATERIAL AND METHODS}

Two studies were conducted using hair ram lambs: Blackbelly, Pelibuey and Blackbelly $x$ Pelibuey (Crossbred). In the first study, the development of the circulating profiles of $\mathrm{LH}$ and $T$ were evaluated by means of serial blood samplings. In the second study, graded doses of $\mathrm{GnRH}$ were administered to evaluate the development of the response, in terms of $\mathrm{LH}$ and $\mathrm{T}$ secretion.

\section{General conditions}

The studies were carried out in the state of Querétaro, México (200 42' N; $100^{\circ}$ 01' W). The experimental protocols and procedures were approved by the Institutional Subcommittee for Caring and Use of Animals for Experimentation (SICUAE), College of Veterinary Medicine and Animal Husbandry, National Autonomous University of Mexico, and according to the "International Guiding Principles for Biomedical Research Involving Animals" (http:// www.cioms.ch/images/stories/CIOMS/ IGP2012.pdf). Ram lambs were born between March 2 and 29 (Study 1) or between October 29 and November 14 (Study 2). Before weaning (70 d of age), lambs were creep fed a diet containing $16.9 \% \mathrm{CP}$ and $2.74 \mathrm{Mcal} \mathrm{ME} / \mathrm{kg}$ DM, and ewes were fed a diet containing $13.04 \% \mathrm{CP}$ and $2.33 \mathrm{Mcal} \mathrm{ME} / \mathrm{kg} \mathrm{DM}$. After weaning, lambs were offered a diet with $14.05 \%$ $\mathrm{CP}$ and $2.65 \mathrm{Mcal} \mathrm{ME} / \mathrm{kg} \mathrm{DM}$, and water ad libitum. One day before each lamb reached 2, $4,8,12,16,20$ and 24 wk of age, body weight (BW) was recorded, scrotal circumference (SC) was measured as described previously(15), and lambs were fitted with indwelling jugular catheters. At that time and at the end of samplings, lambs were administered vitamin B complex (thiamin $0.15 \mathrm{~g}$, riboflavin $0.2 \mathrm{~g}$, nicotinamide $0.3 \mathrm{~g}$, pyridoxine $0.2 \mathrm{~g}$, cyanocobalamin $90 \mu \mathrm{g}$ and D-panthenol $0.15 \mathrm{~g}$ ), to prevent anemia, and a long-acting oxytetracycline solution ( $20 \mathrm{mg} \bullet \mathrm{kg}^{-1} \mathrm{BW}$ ) in a prophylactic manner, as the catheter remained in the vein for at least one day. 
de D-pantenol) para prevenir anemia, y en forma profiláctica, una solución de oxitetraciclina de acción prolongada $\left(20 \mathrm{mg} \bullet \mathrm{kg}^{-1}\right)$, ya que el catéter se mantuvo en la vena durante al menos un día.

Estudio 1. Desarrollo de los perfiles de LH y testosterona

Los corderos se distribuyeron en tres grupos de acuerdo al genotipo, homogéneos con base en el peso al nacimiento, tipo de parto y edad de la madre: Blackbelly $(n=4)$, Pelibuey $(n=5)$; Cruzas $(n=8)$. A las $2,4,8,12,16,20$ y 24 semanas de edad, se obtuvieron muestras de sangre cada 15 min, durante 6 h (0700 a 1300 h), y se centrifugaron a $720 \mathrm{xg}$ durante $15 \mathrm{~min}$ a $4{ }^{\circ} \mathrm{C}$; se obtuvo el plasma y se mantuvo congelado a $-20{ }^{\circ} \mathrm{C}$ hasta su análisis. Las variables evaluadas de los perfiles de $\mathrm{LH}$ y $\mathrm{T}$ fueron las concentraciones promedio $\mathrm{y}$ basal $\left(\mathrm{ng} \bullet \mathrm{ml}^{-1}\right)$, la frecuencia (pulsos•6 $\mathrm{h}^{-1}$ ), la amplitud y la altura de los pulsos $\left(\mathrm{ng} \bullet \mathrm{ml}^{-1}\right)$. La detección de los pulsos se realizó utilizando algoritmos computarizados(16) (software Pulsar, modificado para IBM-PC por JF Gitzen y VD Ramírez, Urbana, IL). Los valores $G(n)(G(n)$ parámetros de corte para determinar si un valor o una serie de valores consecutivos pertenece a un pulso-(16)), utilizados para la $\mathrm{LH}$ fueron: $\mathrm{G}(1)=10.0, \mathrm{G}(2)=2.6, \mathrm{G}(3)=1.9, \mathrm{G}(4)=1.5$ y $\mathrm{G}(5)=1.1$; mientras que para $\mathrm{T}$ fueron: $\mathrm{G}(1)=3.8, \mathrm{G}(2)=2.6, \mathrm{G}(3)=1.9, \mathrm{G}(4)=1.5$ y $\mathrm{G}(5)=1.1$. La desviación estándar de los ensayos utilizados en el algoritmo fue modelada como una función cuadrática de la dosis para cada hormona.

Estudio 2. Desarrollo de la respuesta de LH y testosterona a la administración de GnRH

Plan experimental, administración de GnRH y obtención de muestras de sangre. Los corderos se distribuyeron en tres grupos de acuerdo al genotipo, homogéneos con base en el peso al nacimiento, tipo de parto y edad de la madre: Blackbelly $(n=4)$, Pelibuey $(n=4)$; Cruzas $(n=4)$. Se utilizó la sal de acetato de la secuencia natural de GnRH (Sigma Chemical Co., St Louis,
Study 1. Development of LH and testosterone profiles

Ram lambs were allotted in three homogeneous genotype groups, based on birth weight, type of birth and age of the mother: Blackbelly $(n=4)$, Pelibuey $(n=5)$ and Crossbred $(n=8)$. At 2, 4, $8,12,16,20$ and 24 wk of age, blood samples were collected every 15 min, during 6 h (0700 to $1300 \mathrm{~h}$ ), and centrifuged at $720 \mathrm{xg}$ for 15 min at $4{ }^{\circ} \mathrm{C}$; plasma was obtained and kept frozen at $-20{ }^{\circ} \mathrm{C}$ until assayed. The profile variables evaluated for $\mathrm{LH}$ and $\mathrm{T}$ were mean and basal concentrations $\left(\mathrm{ng} \bullet \mathrm{ml}^{-1}\right)$, frequency (pulses $\bullet \mathrm{h}^{-1}$ ), amplitude and height of the pulses $\left(\mathrm{ng} \bullet \mathrm{ml}^{-1}\right)$. Pulse detection was performed using computer algorithms(16) (Pulsar software, modified for IBM-PC by JF Gitzen and VD Ramirez, Urbana, IL). G(n)-values (G(n): cutoff parameters to determine if one point value or a series of consecutive values belong to a pulse-(16)), for $\mathrm{LH}$ were $\mathrm{G}(1)=10.0, \mathrm{G}(2)=2.6$, $\mathrm{G}(3)=1.9, \mathrm{G}(4)=1.5$ and $\mathrm{G}(5)=1.1$; for $\mathrm{T}$ were $\mathrm{G}(1)=3.8, \mathrm{G}(2)=2.6, \mathrm{G}(3)=1.9, \mathrm{G}(4)=1.5$ and $\mathrm{G}(5)=1.1$. The standard deviation of the assays used in the algorithm was modeled as a quadratic function of the dose for each hormone.

Study 2. Development of LH and testosterone response to GnRH administration

Experimental plan, GnRH administration and blood sample collection. Ram lambs were allotted in three homogeneous genotype groups, based on birth weight, type of birth and age of the mother: Blackbelly $(n=4)$, Pelibuey $(n=4)$ and Crossbred $(n=4)$. The acetate salt of the natural sequence of $\mathrm{GnRH}$ was used (Sigma Chemical Co., St Louis, MO.). At the same ages as in the study 1 , animals were challenged in consecutive days with graded doses of GnRH: 3, 9 and $27 \mathrm{ng} \bullet \mathrm{kg}^{-1}$ BW, via i.v. (small, medium and large dose, respectively), starting with the small dose at each age. The small dose was established based on a previous study with adult rams, where it was calculated that the administration of $3 \mathrm{ng} \bullet \mathrm{kg}^{-1}$ generated LH pulses with characteristics similar to non-induced pulses(17); therefore, it was initially considered 
MO.). En las mismas edades como en el estudio 1 , los animales se desafiaron en días consecutivos con dosis crecientes de GnRH: 3 , 9 y 27 ng・kg-1, vía i.v. (dosis baja, media y alta, respectivamente), comenzando con la dosis baja en cada edad. La dosis baja se estableció con base en un estudio previo con machos adultos, donde se calculó que la administración de $3 \mathrm{ng} \bullet \mathrm{kg}^{-1}$ generaba pulsos de $\mathrm{LH}$ con características similares a los pulsos no inducidos(17); por lo tanto, inicialmente se consideró como la dosis fisiológica. Se obtuvieron muestras de sangre a $-15,0,15$, $30,60,120$ y 180 min de la administración de GnRH (0700 a 1015 h), y se procesaron como se explicó para el Estudio 1.

Análisis de la respuesta hormonal a la administración de GnRH. Las variables de respuesta de LH y T a la administración de GnRH fueron las siguientes: la altura del pico (concentración máxima de la hormonas después de la administración de $\mathrm{GnRH}$ ); el área bajo la curva de respuesta (AUC), la cual se calculó usando la regla trapezoidal a partir de la concentración inicial (promedio de las muestras de -15 y 0 min) hasta $3 \mathrm{~h}$ después de la administración de $\mathrm{GnRH}$; la magnitud de la respuesta (DLH y DT), se calculó restando la concentración inicial a la altura del pico, y usando la concentración inicial como covariable para cada hormona.

\section{Análisis hormonal}

Las concentraciones de $\mathrm{LH}$ se determinaron mediante un RIA de doble anticuerpo(18), la sensibilidad fue de $95 \mathrm{pg} \bullet \mathrm{ml}^{-1}$, los CV intra e inter-ensayo fueron 8.6 y $9.5 \%$, y 14.2 y $7 \%$, para los estudios 1 y 2 , respectivamente. Las concentraciones de testosterona se determinaron mediante un RIA de fase sólida (Coat-A-Count ${ }^{2}$, Siemens Medical Solutions Diagnostic; Los Angeles CA, EE.UU.), la sensibilidad fue de 40 $\mathrm{pg} \bullet \mathrm{ml}^{-1}$, los CV intra e inter-ensayo fueron 4.9 y $7.1 \%$, y 5 y $5.9 \%$, para los estudios 1 y 2 , respectivamente. as the physiological dose. Blood samples were collected at $-15,0,15,30,60,120$ and 180 min from GnRH administration (0700 to 1015 h), and processed as explained for study 1 .

Analysis of the hormonal response to $\mathrm{GnRH}$ administration. The variables of $\mathrm{LH}$ and $\mathrm{T}$ response to $\mathrm{GnRH}$ administration were the following: peak height (the highest hormone concentration subsequent to $\mathrm{GnRH}$ administration); area under the curve of response (AUC), which was calculated using the trapezoid rule from the initial concentration (average of the -15 and 0 min samples) until 3 $\mathrm{h}$ after $\mathrm{GnRH}$ administration; magnitude of the response $(\Delta \mathrm{LH}$ and $\Delta \mathrm{T})$, calculated by subtracting the average initial concentration from the peak height, and using the initial concentration as a covariable for each hormone.

\section{Hormone analysis}

LH concentrations were determined by a double antibody RIA(18), sensitivity was $95 \mathrm{pg} \bullet \mathrm{ml}^{-1}$, the intra- and inter-assay CV were 8.6 and $9.5 \%$, and 14.2 and $7 \%$ for studies 1 and 2, respectively. Testosterone concentrations were determined by a solid phase RIA (Coat-ACount $\AA$, Siemens Medical Solutions Diagnostic; Los Angeles CA, USA); sensitivity was 40 $\mathrm{pg} \bullet \mathrm{ml}^{-1}$; the intra- and inter-assay CV were 4.9 and $7.1 \%$, and 5 and $5.9 \%$ for studies 1 and 2 , respectively.

\section{Statistical analyses}

Hormone data were analyzed as a completely randomized design, using the procedure MIXED of SAS (SAS Institute Inc. Cary NC, USA) for repeated measures, with the statement "repeated", the option sub=animal (genotype) and the autoregressive (1) covariance structure within animal. Genotype, age (and dose in study 2 ) and the corresponding interactions were included in the model. The endocrine variables were transformed to $\log _{n}(Y+1)$, so the assumptions of the analysis of variance were valid; after analysis, data were transformed back to actual values for tables and figures. Data of 


\section{Análisis estadístico}

Los datos hormonales se analizaron como un diseño completamente al azar, utilizando el procedimiento MIXED de SAS (SAS Institute Inc. Cary NC, EE.UU.) para mediciones repetidas, con el comando "repeated", la opción sub= animal (genotipo) y la estructura de la covarianza dentro de animal auto regresiva (1). El genotipo, la edad (y la dosis en el estudio 2) y las interacciones correspondientes se incluyeron en el modelo. Las variables endocrinas fueron transformadas al $\log _{n}(Y+1)$, para cumplir con los supuestos del análisis de varianza; después del análisis, los datos se transformaron a los valores reales para los cuadros y figuras. Los datos de peso y CE de los dos estudios se combinaron antes del análisis, y cada estudio se incluyó en el modelo como bloque. Se consideraron diferencias significativas con valores de $P<0.05$ y tendencia a ser significativas con valores de $P$ entre 0.05 y 0.1 .

\section{RESULTADOS}

Desarrollo de los perfiles de LH

El efecto del genotipo y la interacción genotipo por edad no fueron significativos para ninguna de las variables de los perfiles de $\mathrm{LH}$, mientras que el efecto de la edad sí lo fue para todas las variables (Cuadro 1). La menor concentración promedio se observó en la semana 2 , se detectó una elevación en la semana $4(P<0.05)$, después hubo un aumento progresivo, alcanzando la mayor concentración en la semana 12 para descender en la semana $16(P<0.05)$, con un incremento adicional observado en la semana 24 $(P<0.05)$. La concentración basal no cambió entre 2 y 8 semanas; después aumentó en la semana $12(P<0.05)$, alcanzando una meseta hasta la semana 20 , con un incremento adicional en la semana $24(P<0.05)$. La frecuencia de pulsos fue mayor en la semana 8 , pero no se detectaron diferencias entre 2 y 20 semanas, mientras que la menor frecuencia se detectó en la semana $24(P<0.05)$. Se detectaron pulsos de baja amplitud en la semana 2 , después la
BW and SC were pooled across studies before the analysis, and each study was included in the model as a block. Differences were considered significant with $P<0.05$, and tended to be significant with $P$ values between 0.05 and 0.1 .

\section{RESULTS}

Development of $\mathrm{LH}$ profiles

The effect of genotype and genotype by age interaction were not significant for any of the variables of LH profiles, whilst the effect of age was significant for all variables (Table 1 ). The smallest mean concentration was observed on wk 2, an elevation was detected on wk 4 $(P<0.05)$; then, there was a progressive increase, reaching the greatest concentration on wk 12 to fall on wk $16(P<0.05)$, with an additional increase observed on wk $24(P<0.05)$. Basal concentration did not change from 2 to 8 wk; then, increased at wk $12(P<0.05)$, when reached a plateau until wk 20 , with an additional increase on wk $24(P<0.05)$. Pulse frequency was greatest on wk 8 , but no differences were detected from 2 to $20 \mathrm{wk}$, whilst the least frequency was detected on wk $24(P<0.05)$. Pulses of low amplitude were detected on wk 2; then, amplitude increased drastically on wk 4 $(P<0.05)$ to reach the greatest value on wk 12, with a decreasing trend thereafter.

\section{Development of testosterone profiles}

The effect of genotype and genotype by age interaction were not significant for any of the variables of $\mathrm{T}$ profiles, whilst the effect of age was significant for all variables (Table 1). Mean T concentration increased constantly from wk 2 to $24(P<0.05)$. Basal concentration was maintained low until wk 8; thereafter, a sustained increase was observed from wk 12 to $24(P<0.05)$. Pulses of low magnitude were detected in some animals at 2 wk of age, but the average frequency was very small and was different from the rest of the ages. The highest frequency was observed on wk 12 , but it was not different from wk 8, 16, 20 and 24. Pulse 
Cuadro 1. Medias \pm EE de las variables de los perfiles plasmáticos de LH y testosterona en corderos de pelo de 2 a 24 semanas de edad

Table 1. Means \pm SE for variables of LH and testosterone plasma profiles in hair ram lambs from weeks 2 to 24 of age

\begin{tabular}{|c|c|c|c|c|c|}
\hline Hormone & $\begin{array}{c}\text { Age } \\
\text { (weeks) }\end{array}$ & $\begin{array}{l}\text { Mean concentration } \\
\left(\mathrm{ng} \cdot \mathrm{ml}^{-1}\right)\end{array}$ & $\begin{array}{l}\text { Basal concentration } \\
\left(\mathrm{ng} \cdot \mathrm{ml}^{-1}\right)\end{array}$ & $\begin{array}{l}\text { Pulse frequency } \\
\left.\text { (pulses } 6 h^{-1}\right)\end{array}$ & $\begin{array}{c}\text { Pulse amplitude } \\
\text { (ng・ml-1) }\end{array}$ \\
\hline \multirow[t]{7}{*}{ LH } & 2 & $\mathrm{v} 1.18 \pm 0.24$ & $v_{0} 0.71 \pm 0.18$ & $v w 2.35 \pm 0.33$ & $v_{2} .74 \pm 0.46$ \\
\hline & 4 & $w 2.16 \pm 0.34$ & $v w 0.89 \pm 0.19$ & $v w 2.29 \pm 0.21$ & $w \times 8.02 \pm 1.46$ \\
\hline & 8 & $w \times 2.52 \pm 0.43$ & $v w_{1} .07 \pm 0.23$ & $w_{2} .59 \pm 0.26$ & $w \times 7.31 \pm 1.11$ \\
\hline & 12 & $x y 3.42 \pm 0.35$ & $\times 1.94 \pm 0.31$ & $v w 1.76 \pm 0.32$ & $x 11.11 \pm 1.78$ \\
\hline & 16 & $w 2.33 \pm 0.25$ & $w \times 1.40 \pm 0.20$ & $v w 1.71 \pm 0.21$ & $w \times 7.97 \pm 1.03$ \\
\hline & 20 & $w \times 2.48 \pm 0.27$ & $x 1.76 \pm 0.25$ & $v w 2.06 \pm 0.29$ & $w 6.00 \pm 1.17$ \\
\hline & 24 & У3.77 \pm 0.39 & У2.87 \pm 0.35 & $\mathrm{v} 1.65 \pm 0.24$ & $w \times 6.56 \pm 1.07$ \\
\hline \multirow[t]{7}{*}{ Testosterone } & 2 & $v_{0} 0.07 \pm 0.01$ & $v_{0} 0.04 \pm 0.00$ & $v_{0} 0.40 \pm 0.16$ & $v_{0} 0.69 \pm 0.21$ \\
\hline & 4 & $v w 0.17 \pm 0.03$ & $v_{0} 0.04 \pm 0.00$ & $w_{1} .20 \pm 0.26$ & $\mathrm{v} 1.00 \pm 0.16$ \\
\hline & 8 & $w_{0} 0.33 \pm 0.07$ & $v_{0} 0.09 \pm 0.02$ & $w \times 1.60 \pm 0.21$ & $v_{0} 0.95 \pm 0.15$ \\
\hline & 12 & $\times 0.76 \pm 0.14$ & $w_{0} 0.30 \pm 0.09$ & $\times 2.00 \pm 0.31$ & $\mathrm{v} 1.85 \pm 0.28$ \\
\hline & 16 & $y 1.69 \pm 0.22$ & $w_{0} 0.46 \pm 0.11$ & $w \times 1.87 \pm 0.19$ & $w 4.51 \pm 0.43$ \\
\hline & 20 & y2.01 \pm 0.29 & $x_{0} .70 \pm 0.15$ & $w x 1.60 \pm 0.13$ & $\times 5.13 \pm 0.73$ \\
\hline & 24 & $z 2.31 \pm 0.22$ & $\mathrm{y} 1.03 \pm 0.23$ & $w \times 1.47 \pm 0.17$ & $\times 5.59 \pm 0.48$ \\
\hline
\end{tabular}

vwxyz Means without a common superscript within variable and hormone are different $(P<0.05)$.

amplitud aumentó drásticamente en la semana 4 $(P<0.05)$ para alcanzar el mayor valor en la semana 12, con una tendencia descendente a partir de entonces.

Desarrollo de los perfiles de testosterona

El efecto del genotipo y la interacción genotipo por edad no fueron significativos para ninguna de las variables de los perfiles de T, mientras que el efecto de la edad sí lo fue para todas las variables (Cuadro 1). La concentración promedio de $\mathrm{T}$ aumentó constantemente entre las semanas 2 y $24 \quad(P<0.05)$. La concentración basal se mantuvo baja hasta la semana 8 ; a partir de entonces se observó un aumento sostenido de la semana 12 a $24(P<0.05)$. Se detectaron pulsos de baja magnitud en algunos animales a las 2 semanas de edad, pero la frecuencia era muy baja y diferente del resto de las edades. La frecuencia más alta se observó en la semana 12 , pero no fue diferente de las semanas $8,16,20$ y 24 . La amplitud de los pulsos fue pequeña entre las semanas 2 y 8 , amplitude was small between wk 2 and 8; then, significant increases were observed on wk 16 and $20(P<0.05)$, with no further changes.

Development of LH and testosterone responses to GnRH administration

The effects of the main factors and the corresponding interactions on $\mathrm{LH}$ and $\mathrm{T}$ response to the administration of $\mathrm{GnRH}$ are shown in Table 2.

$\mathrm{LH}$ secretion after $\mathrm{GnRH}$ administration. The triple interaction genotype by age by dose was not significant for any of the variables of $\mathrm{LH}$ response (Table 2). Genotype by age interaction was or tended to be significant for peak height of $\mathrm{LH}$ secretion $(P=0.046)$ and for $\Delta \mathrm{LH}$ $(P=0.063$; Tables 2 and 3$)$. There were not differences between genotypes on wk 2, 4, 16 and 20, whereas on wk 8, 12 and 24, LH response was greatest in Pelibuey lambs. Pelibuey and crossbred lambs reached the highest response on wk $8(P<0.05)$, which was 
Cuadro 2. Efecto de los factores principales y las interacciones respectivas sobre la altura del pico, el área bajo la curva (AUC) y la magnitud de la respuesta (valores $\Delta$ ) de LH y testosterona, después de la administración de $\mathrm{GnRH}$ en corderos de pelo

Table 2. Effect of the main factors and the corresponding interactions on peak height, area under the curve (AUC) and magnitude of the response ( $\Delta$ values) of $\mathrm{LH}$ and testosterone, after administration of $\mathrm{GnRH}$ in hair ram lambs

\begin{tabular}{|c|c|c|c|c|}
\hline Variable & $\mathrm{LH}$ & $P$-value & Testosterone & $P$-value \\
\hline Peak height & $\begin{array}{l}\text { Genotype } \\
\text { Dose } \\
\text { Age } \\
\text { Genotype x Age } \\
\text { Genotype x Dose } \\
\text { Dose x Age } \\
\text { Genotype x Age x Dose }\end{array}$ & $\begin{array}{l}0.002 \\
0.001 \\
0.001 \\
0.046 \\
0.006 \\
0.001 \\
0.458\end{array}$ & $\begin{array}{l}\text { Genotype } \\
\text { Dose } \\
\text { Age } \\
\text { Genotype x Age } \\
\text { Genotype x Dose } \\
\text { Dose x Age } \\
\text { Genotype x Age x Dose }\end{array}$ & $\begin{array}{l}0.105 \\
0.001 \\
0.001 \\
0.425 \\
0.406 \\
0.001 \\
0.664\end{array}$ \\
\hline AUC & $\begin{array}{l}\text { Genotype } \\
\text { Dose } \\
\text { Age } \\
\text { Genotype x Age } \\
\text { Genotype x Dose } \\
\text { Dose x Age } \\
\text { Genotype x Age x Dose }\end{array}$ & $\begin{array}{l}0.012 \\
0.001 \\
0.001 \\
0.465 \\
0.06 \\
0.001 \\
0.207\end{array}$ & $\begin{array}{l}\text { Genotype } \\
\text { Dose } \\
\text { Age } \\
\text { Genotype x Age } \\
\text { Genotype x Dose } \\
\text { Dose x Age } \\
\text { Genotype x Age x Dose }\end{array}$ & $\begin{array}{l}0.080 \\
0.001 \\
0.001 \\
0.644 \\
0.405 \\
0.002 \\
0.676\end{array}$ \\
\hline$\Delta$ Values & $\begin{array}{l}\text { Genotype } \\
\text { Dose } \\
\text { Age } \\
\text { Genotype x Age } \\
\text { Genotype x Dose } \\
\text { Dose x Age } \\
\text { Genotype x Age x Dose }\end{array}$ & $\begin{array}{l}0.002 \\
0.001 \\
0.001 \\
0.063 \\
0.034 \\
0.001 \\
0.444\end{array}$ & $\begin{array}{l}\text { Genotype } \\
\text { Dose } \\
\text { Age } \\
\text { Genotype x Age } \\
\text { Genotype x Dose } \\
\text { Dose x Age } \\
\text { Genotype x Age x Dose }\end{array}$ & $\begin{array}{l}0.082 \\
0.001 \\
0.001 \\
0.712 \\
0.161 \\
0.001 \\
0.647\end{array}$ \\
\hline
\end{tabular}

después se observaron aumentos significativos en las semanas 16 y $20(P<0.05)$, sin más cambios posteriores.

Desarrollo de las respuestas de LH y testosterona a la administración de GnRH Los efectos de los factores principales y las interacciones correspondientes de la respuesta de LH y T a la administración de GnRH se muestran en el Cuadro 2.

Secreción de LH después de la administración de $G n R H$. La triple interacción genotipo por edad por dosis no fue significativa para ninguna de las variables de la respuesta de LH (Cuadro 2). La interacción genotipo por edad fue o tendió a ser significativa para la altura del pico de $\mathrm{LH}$ maintained until wk 16, whereas Blackbelly lambs did not reach the highest response until wk 16 ( $P<0.05)$; after wk 16, peak height showed a decreasing trend for all the genotypes. Genotype by dose interaction was or tended to be significant for peak height $(P=0.006)$, AUC $(P=0.06)$ and $\Delta \mathrm{LH}(P=0.034 ;$ Table 2$)$. No differences between genotypes were detected for the small dose (Table 4; peak height and $\Delta \mathrm{LH})$, but for the medium and large doses, Pelibuey had the highest and Blackbelly the lowest $\mathrm{LH}$ response to $\mathrm{GnRH}$, with crossbred lambs being intermediate. Dose by age interaction was significant for all the variables of $\mathrm{LH}$ response (Table 2); differences between doses were detected at every age in a dosedependent manner (Table 5), except for AUC at 
DESARROLLO DEL EJE REPRODUCTIVO ENDOCRINO EN CORDEROS DE PELO

Cuadro 3. Interacción genotipo por edad sobre la altura del pico y la magnitud de la respuesta de $\mathrm{LH}(\Delta \mathrm{LH})$, después de la administración de $\mathrm{GnRH}$. Los valores son medias $\pm \mathrm{EE}$ $\left(\mathrm{ng} \cdot \mathrm{ml}^{-1}\right)$

Table 3. Genotype by age interaction on peak height and magnitude of the $\mathrm{LH}$ response $(\Delta \mathrm{LH})$, after administration of $\mathrm{GnRH}$. Values are means $\pm \mathrm{SE}\left(\mathrm{ng} \cdot \mathrm{ml}^{-1}\right)$

\begin{tabular}{|c|c|c|c|c|}
\hline \multirow[b]{2}{*}{ Variable } & \multirow{2}{*}{$\begin{array}{c}\text { Age } \\
\text { (weeks) }\end{array}$} & \multicolumn{3}{|c|}{ Genotype } \\
\hline & & Blackbelly & Crossbred & Pelibuey \\
\hline \multirow[t]{7}{*}{ Peak height } & 2 & $w_{3} .59 \pm 0.46^{a}$ & w3.07 $\pm 0.63^{a}$ & $w_{4} .56 \pm 0.81^{a}$ \\
\hline & 4 & $w \times 5.86 \pm 1.25^{a}$ & $w \times 4.70 \pm 1.02^{a}$ & w5.35 $\pm 1.19 \mathrm{a}$ \\
\hline & 8 & $w \times 6.34 \pm 1.61 a$ & $z 12.80 \pm 3.33^{b}$ & $\mathrm{y} 16.86 \pm 3.69 \mathrm{~b}$ \\
\hline & 12 & $\times 6.71 \pm 1.18^{a}$ & y $8.42 \pm 1.53^{a}$ & $\mathrm{y} 14.15 \pm 2.15^{\mathrm{b}}$ \\
\hline & 16 & $\mathrm{y} 10.97 \pm 2.06 \mathrm{a}$ & $\mathrm{z} 12.68 \pm 1.92^{\mathrm{a}}$ & $\mathrm{y} 15.64 \pm 2.82^{\mathrm{a}}$ \\
\hline & 20 & $x y 7.40 \pm 1.04 a$ & $y 6.94 \pm 1.24^{a}$ & $\mathrm{x} 10.16 \pm 1.80^{a}$ \\
\hline & 24 & $x y 7.10 \pm 0.97 a b$ & $x y 6.45 \pm 1.03^{a}$ & $x y 11.63 \pm 2.29 b$ \\
\hline \multirow[t]{7}{*}{$\Delta \mathrm{LH}$} & 2 & w2.86 $\pm 0.48^{a}$ & w2.39 $\pm 0.63 a$ & w3. $77 \pm 0.77 a$ \\
\hline & 4 & $w \times 4.29 \pm 1.27 a$ & $w \times 4.11 \pm 0.98^{a}$ & $w_{4} 4.4 \pm 1.24^{a}$ \\
\hline & 8 & $w \times 4.99 \pm 1.6^{a}$ & $z 11.23 \pm 3.25^{b}$ & $\mathrm{y} 14.9 \pm 3.78^{b}$ \\
\hline & 12 & $w \times 4.59 \pm 1.06^{a}$ & $\mathrm{y}_{5.82} \pm 1.68 \mathrm{a}$ & $\mathrm{y} 11.12 \pm 2.38 \mathrm{~b}$ \\
\hline & 16 & $y 8.53 \pm 2.06^{a}$ & $\mathrm{z} 10.25 \pm 1.83^{\mathrm{a}}$ & $\mathrm{y} 12.95 \pm 2.83^{\mathrm{a}}$ \\
\hline & 20 & $x y 5.45 \pm 1.05^{a}$ & xy5.76 $\pm 1.26 a$ & x8.87 $\pm 1.8 \mathrm{a}$ \\
\hline & 24 & $\times 5.04 \pm 1.05^{a}$ & $x y 5.71 \pm 1.08^{a}$ & $x y 10.59 \pm 2.14^{b}$ \\
\hline
\end{tabular}

ab Means without a common superscript within variable and age are different $(P<0.05)$.

wxyz Means without a common superscript within variable and genotype are different $(P<0.05)$.

Cuadro 4. Interacción genotipo por dosis de $\mathrm{GnRH}$ sobre la altura del pico $\left(\mathrm{ng} \cdot \mathrm{ml}^{-1}\right)$, el área bajo la curva de respuesta (AUC; $n g \cdot \mathrm{ml}^{-1} \cdot 3 \mathrm{~h}^{-1}$ ) y la magnitud de la respuesta de LH (Delta LH; $\mathrm{ng} \cdot \mathrm{ml}^{-1}$ ), después de la administración de $\mathrm{GnRH}$. (Medias $\pm \mathrm{EE}$ )

Table 4. Genotype by $\mathrm{GnRH}$ dose interaction on peak height $\left(\mathrm{ng}^{\circ} \mathrm{ml}^{-1}\right)$, area under the curve of response (AUC; $\mathrm{ng}^{\circ} \mathrm{ml}^{-1} \cdot 3 \mathrm{~h}^{-1}$ ) and magnitude of the $\mathrm{LH}$ response (Delta $\mathrm{LH} ; \mathrm{ng} \cdot \mathrm{ml}^{-1}$ ), after administration of $\mathrm{GnRH}$. (Means $\pm \mathrm{SE}$ )

\begin{tabular}{lcccc}
\hline \multirow{2}{*}{ Variable } & $\begin{array}{c}\text { GnRH dose } \\
\left(\mathrm{ng}^{*} \mathrm{~kg}^{-1} \mathrm{BW}\right)\end{array}$ & Blackbelly & Crossbred & Pelibuey \\
\cline { 2 - 5 } Peak height & 3 & $2.96 \pm 0.37^{\mathrm{a}}$ & $2.76 \pm 0.41^{\mathrm{a}}$ & $3.15 \pm 0.40^{\mathrm{a}}$ \\
& 9 & $7.14 \pm 0.70^{\mathrm{a}}$ & $8.09 \pm 0.82^{\mathrm{a}}$ & $10.89 \pm 1.06^{\mathrm{b}}$ \\
& 27 & $10.56 \pm 0.97^{\mathrm{a}}$ & $12.20 \pm 1.34^{\mathrm{a}}$ & $19.04 \pm 1.62^{\mathrm{b}}$ \\
AUC & 3 & $316 \pm 35^{\mathrm{a}}$ & $284 \pm 44^{\mathrm{ab}}$ & $338 \pm 34^{\mathrm{b}}$ \\
& 9 & $488 \pm 50^{\mathrm{a}}$ & $497 \pm 40^{\mathrm{ab}}$ & $666 \pm 67^{\mathrm{b}}$ \\
& 27 & $665 \pm 64^{\mathrm{a}}$ & $723 \pm 65^{\mathrm{ab}}$ & $1064 \pm 85^{\mathrm{b}}$ \\
$\Delta \mathrm{LH}$ & 3 & $1.16 \pm 0.23^{\mathrm{a}}$ & $1.51 \pm 0.33^{\mathrm{a}}$ & $1.73 \pm 0.37 \mathrm{a}$ \\
& 9 & $5.34 \pm 0.67^{\mathrm{a}}$ & $6.59 \pm 0.77^{\mathrm{ab}}$ & $9.06 \pm 0.97^{\mathrm{b}}$ \\
& 27 & $8.84 \pm 0.94^{\mathrm{a}}$ & $10.78 \pm 1.26^{\mathrm{a}}$ & $17.27 \pm 1.63^{\mathrm{b}}$ \\
\hline
\end{tabular}

ab Means without a common superscript within variable and dose are different $(P<0.05)$. 
$(P=0.046)$ y para $\Delta \mathrm{LH}(P=0.063$; Cuadros 2 y $3)$. No hubo diferencias entre los genotipos en las semanas $2,4,16$ y 20 , mientras que en las semanas 8,12 y 24, la respuesta de LH fue mayor en los corderos Pelibuey. Los corderos Pelibuey y cruzados alcanzaron la mayor respuesta en la semana $8(P<0.05)$, la cual se mantuvo hasta la semana 16 , mientras que los corderos Blackbelly alcanzaron la mayor respuesta hasta la semana $16(P<0.05)$; después de la semana 16, la altura del pico mostró una tendencia decreciente en todos los wk 2. The effects of genotype, GnRH dose and age on the three variables of LH response were significant (Table 2). Pelibuey ram lambs always showed the greatest response, as compared with Blackbelly and crossbred lambs (Table 6). The response to GnRH administration was in a dose-dependent manner (Table 6). Regarding the effect of age, the greatest $\mathrm{LH}$ response was observed between 8 and $16 \mathrm{wk}$ of age; thereafter, the response decreased $(P<0.05)$ for the three variables evaluated (Figure 1).

Cuadro 5. Interacción dosis por edad sobre la altura del pico $\left(\mathrm{ng}^{\bullet} \mathrm{ml}^{-1}\right)$, el área bajo la curva de respuesta $\left(A \cup C ; n g \cdot \mathrm{ml}^{-1} \cdot 3 \mathrm{~h}^{-1}\right)$ y la magnitud de la respuesta de $\mathrm{LH}\left(\Delta \mathrm{LH} ; \mathrm{ng}^{\circ} \mathrm{ml}^{-1}\right)$, después de la administración de $\mathrm{GnRH}$, (Medias $\pm \mathrm{EE}$ )

Table 5. Dose by age interaction on peak height $\left(\mathrm{ng} \cdot \mathrm{ml}^{-1}\right)$, area under the curve of response (AUC; $\left.\mathrm{ng} \cdot \mathrm{ml}^{-1} \cdot 3 \mathrm{~h}^{-1}\right)$ and magnitude of the $\mathrm{LH}$ response $\left(\Delta \mathrm{LH} ; \mathrm{ng} \cdot \mathrm{ml}^{-1}\right)$, after administration of $\mathrm{GnRH}$. (Means $\pm \mathrm{SE}$ )

\begin{tabular}{|c|c|c|c|c|}
\hline \multirow[b]{2}{*}{ Variable } & \multirow[b]{2}{*}{ Age(weeks) } & \multicolumn{3}{|c|}{ Doses of $\mathrm{GnRH}$} \\
\hline & & $3 \mathrm{ng} \cdot \mathrm{kg}^{-1} \mathrm{BW}$ & $9 \mathrm{ng} \cdot \mathrm{kg}^{-1} \mathrm{BW}$ & $27 \mathrm{ng}^{\circ} \mathrm{kg}^{-1} \mathrm{BW}$ \\
\hline Peak & 2 & $w \times 2.36 \pm 0.60 a$ & w3.33 $\pm 0.34 b$ & w5.68 $\pm 0.67 c$ \\
\hline \multirow[t]{6}{*}{ height } & 4 & w2.08 $\pm 0.70^{a}$ & $w 4.64 \pm 0.84^{b}$ & $x 9.33 \pm 0.98 c$ \\
\hline & 8 & $w \times 2.33 \pm 0.37 a$ & $\mathrm{x} 11.81 \pm 1.73^{b}$ & $\mathrm{z} 23.31 \pm 3.10 \mathrm{c}$ \\
\hline & 12 & $y 4.59 \pm 0.75^{a}$ & $\mathrm{x} 10.58 \pm 1.31^{b}$ & $\mathrm{y} 14.40 \pm 2.25^{\mathrm{c}}$ \\
\hline & 16 & У3.95 $\pm 0.53^{\mathrm{a}}$ & $\mathrm{y} 14.43 \pm 1.34^{b}$ & $z 21.00 \pm 2.06 c$ \\
\hline & 20 & $x 2.61 \pm 0.32^{a}$ & $x 8.73 \pm 0.71 b$ & $\mathrm{y} 13.42 \pm 1.21^{\mathrm{c}}$ \\
\hline & 24 & $x 2.68 \pm 0.38^{a}$ & $x 8.73 \pm 0.76^{b}$ & $\mathrm{y} 14.18 \pm 1.83 \mathrm{c}$ \\
\hline \multirow[t]{7}{*}{ AUC } & 2 & $w_{2} 52 \pm 47^{a}$ & $w_{222} \pm 22^{a}$ & $w 344 \pm 36^{b}$ \\
\hline & 4 & $w 237 \pm 38 a$ & $w_{2} 28 \pm 36^{b}$ & $\times 501 \pm 48 c$ \\
\hline & 8 & $w \times 290 \pm 34^{a}$ & $x y 722 \pm 116^{b}$ & $z 1184 \pm 131^{c}$ \\
\hline & 12 & y527 $\pm 77 a$ & $x y 720 \pm 104 b$ & y868 $\pm 116^{b}$ \\
\hline & 16 & xy391 $\pm 46^{a}$ & $y 815 \pm 65^{b}$ & $\mathrm{z} 1153 \pm 92^{c}$ \\
\hline & 20 & $w_{243} \pm 49 a$ & $x 595 \pm 51 b$ & $y z 893 \pm 90^{c}$ \\
\hline & 24 & $\mathrm{w}_{256} \pm 42^{\mathrm{a}}$ & $x 562 \pm 54 b$ & $y z 940 \pm 129 c$ \\
\hline \multirow[t]{7}{*}{$\Delta \mathrm{LH}$} & 2 & $w \times 1.54 \pm 0.57 a$ & $w_{2} .67 \pm 0.30^{b}$ & $w 4.95 \pm 0.64 c$ \\
\hline & 4 & w0.67 $\pm 0.18^{a}$ & w3.67 $\pm 0.93^{b}$ & $x 8.50 \pm 1.02^{c}$ \\
\hline & 8 & $w_{0} 0.90 \pm 0.21^{a}$ & $x 9.72 \pm 1.59 b$ & $z 21.85 \pm 3.15^{c}$ \\
\hline & 12 & y2.52 $\pm 0.79 a$ & $x 7.92 \pm 1.40 b$ & $x y 11.37 \pm 2.48 b$ \\
\hline & 16 & $x y 1.72 \pm 0.48^{a}$ & $\mathrm{y} 11.64 \pm 1.38^{b}$ & $z 18.43 \pm 2.09 c$ \\
\hline & 20 & $w \times 1.21 \pm 0.22^{a}$ & $x 7.11 \pm 0.78 b$ & $\mathrm{y} 11.96 \pm 1.26 \mathrm{C}$ \\
\hline & 24 & $w x 1.55 \pm 0.37 a$ & $x 7.34 \pm 0.90 b$ & $\mathrm{y} 12.76 \pm 1.77 \mathrm{c}$ \\
\hline
\end{tabular}

abc Means without a common superscript within variable and age are different $(P<0.05)$.

wxyz Means without a common superscript within variable and dose are different $(P<0.05)$. 
Cuadro 6. Efecto del genotipo y la dosis de $\mathrm{GnRH}$ sobre la altura del pico $\left(\mathrm{ng} \cdot \mathrm{ml}^{-1}\right)$, el área bajo la curva de respuesta (AUC; ng•ml-1•3h-1) y la magnitud de la respuesta de $\mathrm{LH}(\Delta \mathrm{LH})$ y testosterona $(\Delta \mathrm{T})\left(\mathrm{ng} \cdot \mathrm{ml}^{-1}\right)$, después de la administración de $\mathrm{GnRH}$ (Medias $\pm \mathrm{EE}$ )

Table 6. Effects of genotype and $\mathrm{GnRH}$ dose on peak height $\left(\mathrm{ng} \cdot \mathrm{ml}^{-1}\right)$, area under the curve of response (AUC; $\mathrm{ng}^{\circ} \mathrm{ml}^{-1}$ $\left.\cdot 3 \mathrm{~h}^{-1}\right)$ and magnitude of the $\mathrm{LH}$ and testosterone $(\mathrm{T})$ responses $\left(\Delta ; \mathrm{ng}^{\circ} \mathrm{ml}^{-1}\right)$, after administration of $\mathrm{GnRH}$ (Means \pm SE)

\begin{tabular}{ccccccccc}
\hline & & \multicolumn{3}{c}{ Genotype } & & \multicolumn{3}{c}{ GnRH dose $\left(\mathrm{ng} \cdot \mathrm{kg}^{-1} \mathrm{BW}\right)$} \\
\cline { 3 - 4 } \cline { 7 - 8 } Hormone & Variable & Blackbelly & Crossbred & Pelibuey & & 3 & 9 & 27 \\
\hline LH & Peak height & $6.9 \pm 0.5^{\mathrm{a}}$ & $7.7 \pm 0.7^{\mathrm{a}}$ & $11 \pm 0.9^{\mathrm{b}}$ & & $\mathrm{x} 3 \pm 0.2$ & $\mathrm{y} 8.8 \pm 0.5$ & $\mathrm{z} 14.1 \pm 0.9$ \\
& AUC & $489 \pm 32^{\mathrm{a}}$ & $501 \pm 35^{\mathrm{a}}$ & $689 \pm 48^{\mathrm{b}}$ & & $\mathrm{x} 315 \pm 2$ & $\mathrm{y} 555 \pm 33$ & $\mathrm{z} 826 \pm 46$ \\
& $\Delta \mathrm{LH}$ & $5.1 \pm 0.5^{\mathrm{a}}$ & $6.3 \pm 0.7^{\mathrm{a}}$ & $9.4 \pm 0.9^{\mathrm{b}}$ & & $\mathrm{x} 1.5 \pm 0.2$ & $\mathrm{y} 7.0 \pm 0.5$ & $\mathrm{z} 12.4 \pm 0.9$ \\
$\mathrm{~T}$ & Peak height & $3.1 \pm 0.4^{\mathrm{a}}$ & $3.6 \pm 0.3^{\mathrm{b}}$ & $3.4 \pm 0.4^{\mathrm{ab}}$ & & $\mathrm{y} 2.3 \pm 0.3$ & $\mathrm{z} 3.7 \pm 0.4$ & $\mathrm{z} 3.8 \pm 0.4$ \\
& AUC & $232 \pm 28^{\mathrm{a}}$ & $282 \pm 29^{\mathrm{b}}$ & $283 \pm 36^{\mathrm{b}}$ & & $\mathrm{x} 136 \pm 16$ & $\mathrm{y} 288 \pm 29$ & $\mathrm{z} 372 \pm 39$ \\
& $\Delta \mathrm{T}$ & $1.5 \pm 0.2^{\mathrm{a}}$ & $2 \pm 0.3^{\mathrm{b}}$ & $1.9 \pm 0.3^{\mathrm{ab}}$ & & $\mathrm{y} 0.91 \pm 0.22$ & $\mathrm{z} 2.11 \pm 0.26$ & $\mathrm{z} 2.36 \pm 0.28$ \\
\hline
\end{tabular}

ab Means without a common superscript within hormone and variable are different $(P<0.05)$. xyz Means without a common superscript within hormone and variable are different $(P<0.05)$.

genotipos. La interacción genotipo por dosis fue o tendió a ser significativa para la altura del pico $(P=0.006)$, AUC $(P=0.06)$ y $\Delta \mathrm{LH}(P=0.034$; Cuadro 2). No se detectaron diferencias entre genotipos con la dosis baja (Cuadro 4; altura del pico y $\Delta \mathrm{LH}$ ), pero con las dosis media $y$ alta, Pelibuey tuvo la mayor respuesta y Blackbelly la menor respuesta a $\mathrm{GnRH}$, con los corderos cruzados siendo intermedios. La interacción dosis por edad fue significativa para todas las variables de respuesta de LH (Cuadro $2)$; Se detectaron diferencias entre dosis en cada edad en una forma dosis dependiente (Cuadro 5), excepto para AUC en la semana 2. Los efectos de genotipo, dosis de GnRH y edad sobre las tres variables de respuesta de LH fueron significativos (Cuadro 2). Los corderos Pelibuey siempre mostraron la mayor respuesta, en comparación con los corderos Blackbelly y cruzados (Cuadro 6). La respuesta a la administración de GnRH fue de manera dosis dependiente. Respecto a la edad, la mayor respuesta de LH se observó entre las 8 y 16 semanas de edad; a partir de entonces, la respuesta disminuyó $(P<0.05)$ para las tres variables evaluadas (Figura 1).
Testosterone secretion after GnRH administration. The triple interaction of genotype by age by dose, as well as the interactions of genotype by age and genotype by dose were not significant for any of the variables of $T$ response (Table 2). Dose by age interaction was significant for the three variables of $T$ response (Table 2). No differences among doses (Table 7) were detected for peak height (2 and $4 \mathrm{wk}$ ) and $\Delta \mathrm{T}$ ( $2 \mathrm{wk}$ ); AUC was greater for the large dose, as compared with the small dose (2 wk); AUC and $\Delta \mathrm{T}$ were greater in the large dose, as compared with the other doses. From wk 8 onwards, $\mathrm{T}$ response to the small dose was lower than $T$ response to the medium and large doses, with no difference between the last for all variables, except for AUC at wk 24, when the response to all doses was different from each other.

Within dose, $\mathrm{T}$ response increased as the lambs were older (Table 7); however, differences were not always significant. For the small dose, peak height did not change from 2 to 12 wk of age, but then it increased until wk 24; for the medium and large doses, peak height was not different between 2 and 4 wk, but from wk 8 it increased constantly until the end of the study. Regarding 
Secreción de testosterona después de la administración de GnRH. La triple interacción genotipo por edad por dosis, así como las interacciones de genotipo por edad y genotipo por dosis no fueron significativas para ninguna de las variables de la respuesta de $\mathrm{T}$ (Cuadro 2), mientras que la interacción dosis por edad fue significativa para las tres variables (Cuadro 2). No se detectaron diferencias entre las dosis (Cuadro 7) en la altura del pico (semanas 2 y 4) y $\Delta T$ (semana 2); AUC fue mayor con la dosis alta, en comparación con la dosis baja (semana 2); AUC y $\Delta \mathrm{T}$ fueron mayores con la dosis alta, en comparación con las otras dosis. A partir de la semana 8 , la respuesta de $T$ a la dosis baja fue menor que la respuesta a las dosis media y alta, sin diferencias entre las últimas para todas las variables, excepto para AUC en la semana 24 , cuando la respuesta a todas las dosis fue diferente entre sí.

Dentro de dosis, la respuesta de T aumentó a medida que los corderos eran mayores (Cuadro 7); sin embargo, las diferencias no siempre fueron significativas. Para la dosis baja, la altura del pico no cambió entre 2 y 12 semanas de edad, pero después aumentó hasta la semana 24; para las dosis media y alta, la altura del pico no fue diferente entre las semanas 2 y 4 , pero desde la semana 8 aumentó constantemente hasta el final del estudio. Con respecto al AUC, cuando se utilizaron las dosis baja y media, no hubo diferencia entre las semanas 2 y 4 , después aumentó entre las semanas 8 y 20; para la dosis alta, el AUC aumentó continuamente entre las semanas 2 y 24. $\Delta \mathrm{T}$ no fue diferente entre 2 y 4 semanas, pero desde la semana 8 en adelante aumentó hasta la semana 24 , para todas las dosis.

El efecto del genotipo en las tres variables de la respuesta de $\mathrm{T}$ tendió a ser significativo (Cuadro 2), los corderos Blackbelly siempre mostraron una respuesta inferior a los corderos cruzados (Cuadro 6), con los Pelibuey siendo intermedios. Los efectos de la dosis de GnRH y la edad fueron significativas (Cuadro 2); la
AUC, when the small and medium doses were used, there was no difference between 2 and $4 \mathrm{wk}$, then it increased between wk 8 and 20; the AUC to the large dose increased continuously from 2 to $24 \mathrm{wk}$. $\Delta \mathrm{T}$ was no different between

Figura 1. Efecto de la edad sobre la altura del pico (panel A), el área bajo la curva de respuesta (panel B) y la magnitud de la respuesta (panel C) de LH y testosterona, después de la administración de $\mathrm{GnRH}$

Figure 1. Effect of age on peak height (panel A), area under the curve of response (panel B) and magnitude of the response (panel $\mathrm{C}$ ) of $\mathrm{LH}$ and testosterone, after administration of $\mathrm{GnRH}$
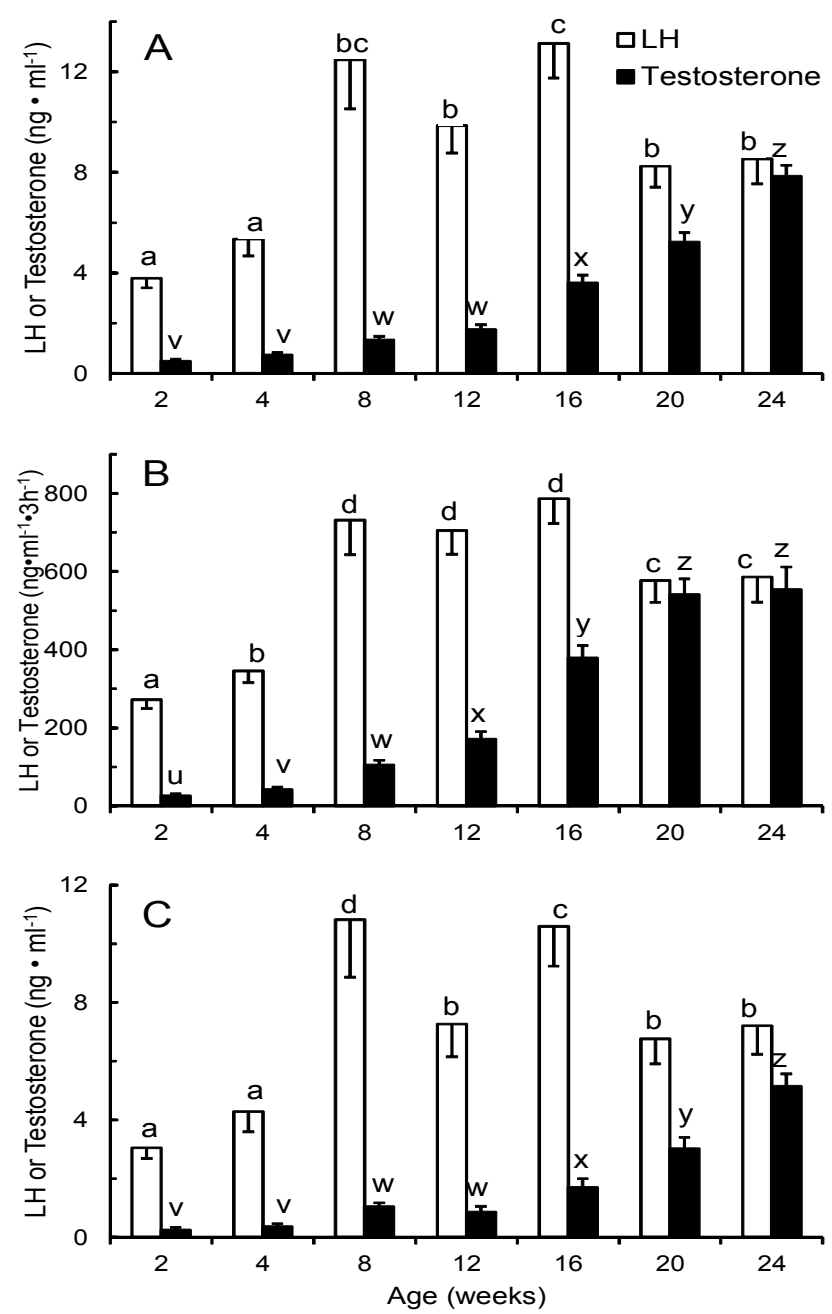

Values are means \pm SE, pooled across genotypes and doses. Means without a common superscript within each panel and hormone (a,b,c,d LH; u,v,w,x,y,z testosterone) indicate differences between ages $(P<0.05)$. 
DESARROLLO DEL EJE REPRODUCTIVO ENDOCRINO EN CORDEROS DE PELO

Cuadro 7. Interacción dosis por edad sobre la altura del pico $\left(\mathrm{ng} \bullet \mathrm{ml}^{-1}\right)$, el área bajo la curva de respuesta (AUC; $\mathrm{ng}^{\circ} \mathrm{ml}^{-1} \cdot 3 \mathrm{~h}^{-1}$ ) y la magnitud de la respuesta de testosterona $\left(\Delta \mathrm{T} ; \mathrm{ng} \cdot \mathrm{ml}^{-}\right.$ $\left.{ }^{1}\right)$, después de la administración de $\mathrm{GnRH}$ (Medias $\pm \mathrm{EE}$ )

Table 7. Dose by age interaction on peak height $\left(\mathrm{ng} \cdot \mathrm{ml}^{-1}\right)$, area under the curve of response (AUC; $\left.\mathrm{ng} \cdot \mathrm{ml}^{-1} \cdot 3 \mathrm{~h}^{-1}\right)$ and magnitude of the testosterone response $\left(\Delta \mathrm{T} ; \mathrm{ng}^{\circ} \mathrm{ml}^{-1}\right)$, after administration of $\mathrm{GnRH}$ (Means $\pm \mathrm{SE}$ )

\begin{tabular}{|c|c|c|c|c|}
\hline \multirow[b]{2}{*}{ Variable } & \multirow{2}{*}{$\begin{array}{c}\text { Age } \\
\text { (weeks) }\end{array}$} & \multicolumn{3}{|c|}{ Doses of $\mathrm{GnRH}$} \\
\hline & & $3 \mathrm{ng} \cdot \mathrm{kg}^{-1} \mathrm{BW}$ & $9 \mathrm{ng}^{\circ} \mathrm{kg}^{-1} \mathrm{BW}$ & $27 \mathrm{ng} \cdot \mathrm{kg}^{-1} \mathrm{BW}$ \\
\hline \multirow[t]{7}{*}{ Peak height } & 2 & $v_{0} 0.46 \pm 0.21^{a}$ & $v_{0.43} \pm 0.10^{a}$ & $v_{0} 0.55 \pm 0.15^{a}$ \\
\hline & 4 & $v_{0} .43 \pm 0.19 a$ & $v_{0.59} \pm 0.17^{a}$ & $v_{0} 0.95 \pm 0.14^{a}$ \\
\hline & 8 & $v_{0} 0.75 \pm 0.24 a$ & $w 1.33 \pm 0.13^{b}$ & $w 1.80 \pm 0.19 b$ \\
\hline & 12 & $\mathrm{v} 1.00 \pm 0.17 \mathrm{a}$ & $w_{2} .08 \pm 0.40^{b}$ & $w_{2} .11 \pm 0.28 b$ \\
\hline & 16 & $w 1.92 \pm 0.45^{a}$ & $\mathrm{x} 4.44 \pm 0.42^{\mathrm{b}}$ & $x 4.45 \pm 0.35^{b}$ \\
\hline & 20 & $x 2.73 \pm 0.51^{a}$ & $y 6.19 \pm 0.37 \mathrm{~b}$ & $y 6.76 \pm 0.37^{b}$ \\
\hline & 24 & y5.72 $\pm 0.86^{a}$ & $\mathrm{z} 8.64 \pm 0.50^{b}$ & $\mathrm{z} 9.19 \pm 0.40^{b}$ \\
\hline \multirow[t]{7}{*}{ AUC } & 2 & $v_{20} \pm 6 a$ & $\mathrm{v} 24 \pm 5^{a b}$ & $\mathrm{v} 36 \pm 12^{b}$ \\
\hline & 4 & $\mathrm{v}_{20} \pm 5^{\mathrm{a}}$ & $\mathrm{v} 30 \pm 9 \mathrm{a}$ & $w 77 \pm 12^{b}$ \\
\hline & 8 & $w 53 \pm 11 a$ & $w 114 \pm 15^{b}$ & $\mathrm{x} 149 \pm 22^{b}$ \\
\hline & 12 & $\mathrm{x} 105 \pm 19^{a}$ & $w 194 \pm 36 b$ & $x 215 \pm 36^{b}$ \\
\hline & 16 & $\mathrm{y} 199 \pm 36 \mathrm{a}$ & $x 439 \pm 45^{b}$ & $y 498 \pm 47 b$ \\
\hline & 20 & $y 276 \pm 47 a$ & $x 619 \pm 29 b$ & $z 729 \pm 39 b$ \\
\hline & 24 & $\mathrm{y} 260 \pm 49 \mathrm{a}$ & $x 556 \pm 60^{b}$ & $z 845 \pm 104 c$ \\
\hline \multirow[t]{7}{*}{$\Delta \mathrm{T}$} & 2 & $v_{0} 0.07 \pm 0.06 a$ & $v_{0} .26 \pm 0.08^{a}$ & $v_{0} .43 \pm 0.14^{a}$ \\
\hline & 4 & $v_{0.02} \pm 0.02^{a}$ & $v_{0} .28 \pm 0.13 a$ & $v_{0} .78 \pm 0.11 b$ \\
\hline & 8 & $w_{0} 0.30 \pm 0.14^{a}$ & $w_{1} .25 \pm 0.13 b$ & $w 1.57 \pm 0.19 b$ \\
\hline & 12 & $w_{0} .33 \pm 0.09 a$ & $\mathrm{w} 1.04 \pm 0.30^{b}$ & $w 1.21 \pm 0.31 b$ \\
\hline & 16 & $x 0.63 \pm 0.24^{a}$ & $x 2.38 \pm 0.44^{b}$ & $x 2.07 \pm 0.30^{b}$ \\
\hline & 20 & $\mathrm{y} 1.11 \pm 0.55^{\mathrm{a}}$ & $y 3.82 \pm 0.56^{b}$ & ${ }^{y} 4.12 \pm 0.70^{b}$ \\
\hline & 24 & $\mathrm{z} 3.72 \pm 1.02^{\mathrm{a}}$ & $25.55 \pm 0.78^{b}$ & $\mathrm{z} 6.16 \pm 0.82^{b}$ \\
\hline
\end{tabular}

ab Means without a common superscript within variable and age are different $(P<0.05)$.

vwxyz Means without a common superscript within variable and dose are different $(P<0.05)$.

respuesta de $\mathrm{T}$ a la dosis baja fue menor que la respuesta a las dosis media y alta (Cuadro $6)$, sin diferencias entre las dosis mayores (altura del pico y $\Delta \mathrm{T}$ ); el AUC fue diferente entre las tres dosis administradas. Las tres variables de la respuesta de $T$ aumentaron a medida que los corderos iban creciendo (Figura 1).

\section{Peso corporal y circunferencia escrotal}

La interacción del genotipo por edad sobre el peso fue significativa ( $P=0.01$; Figura $2 A)$; de
2 and 4 wk, but from wk 8 onwards it increased until wk 24, for all doses.

The effect of genotype on the three variables of $T$ response tended to be significant (Table 2), Blackbelly ram lambs always showed a lower response than crossbred lambs (Table 6), with Pelibuey being intermediate. The effects of $\mathrm{GnRH}$ dose and age were significant (Table 2); T response to the small dose was lower than the response to the medium and large doses $(P<0.05$; Table 6$)$, with no difference between 
la semana 12 en adelante generalmente los corderos Blackbelly fueron más ligeros, mientras que los cruzados fueron más pesados $(P<0.05)$. El efecto del genotipo y la interacción genotipo por edad sobre la CE no fueron significativos. El efecto de la edad sí lo fue $(P<0.001)$, ya que la $\mathrm{CE}$ aumentó conforme los corderos iban creciendo (Figura 2B).

\section{DISCUSIÓN}

Estudios previos han encontrado una relación entre los cambios endocrinos durante el desarrollo prepuberal y algunas características reproductivas en los ovinos. La edad en la que la concentración de LH empieza a aumentar parece estar relacionada con la precocidad y prolificidad de la raza, ya que el aumento de LH se observa más temprano en razas más precoces( 1 y y más prolíficas $(5,19)$. Por lo tanto, la falta de diferencias entre genotipos para cualquiera de las variables de los perfiles de $\mathrm{LH}$ y $T$ fue inesperada, ya que varios autores han encontrado que Blackbelly es una raza más precoz y más prolífica que Pelibuey $(7,8,10,12)$. Es posible que las diferencias entre sexos en los mecanismos endocrinos que conducen a la pubertad(20) hayan ocultado diferencias sutiles entre los machos de estas razas. Alternativamente, como las razas utilizadas en el presente estudio tienen orígenes parecidos( 6$)$, éstas no son muy diferentes entre sí, lo que contribuiría a un desarrollo endocrino similar.

La concentración promedio de $\mathrm{LH}$ siguió un patrón similar a lo reportado previamente para corderos Blackbelly(14) y varias razas de lana $(1,2,3,21,22)$. Se observaron concentraciones bajas durante las primeras semanas de vida( $(2,3)$, alcanzando concentraciones máximas entre las 11 y 13 semanas de edad, dependiendo de la raza $(3,22)$, seguido por una disminución o variaciones mínimas a partir de entonces. En el presente estudio, se detectaron pulsos de baja amplitud desde las 2 semanas de edad, lo cual coincide con lo reportado para razas de lana(1,21), lo que indica que, aunque inmaduro, existe un eje reproductivo funcional a edades the larger doses (peak height and $\Delta \mathrm{T}$ ); AUC was different among the three doses administered $(P<0.05)$. The response of $\mathrm{T}$ increased as the lambs were older for the three variables evaluated (Figure 1).

\section{Body weight and scrotal circumference}

The interaction of genotype by age on BW was significant $(P=0.01$; Figure $2 A)$; from wk 12 onwards, Blackbelly lambs had usually the lightest and crossbred lambs had the heaviest BW $(P<0.05)$. Regarding SC, the effect of the

Figura 2. Medias \pm EE del peso corporal (panel A) y la circunferencia escrotal (panel B) de corderos Blackbelly, Pelibuey y sus cruzas, de 2 a 24 semanas de edad

Figure 2. Mean \pm SE of body weight (panel A) and scrotal circumference (panel B) of Blackbelly and Pelibuey ram lambs and their crosses, from 2 to 24 wk of age
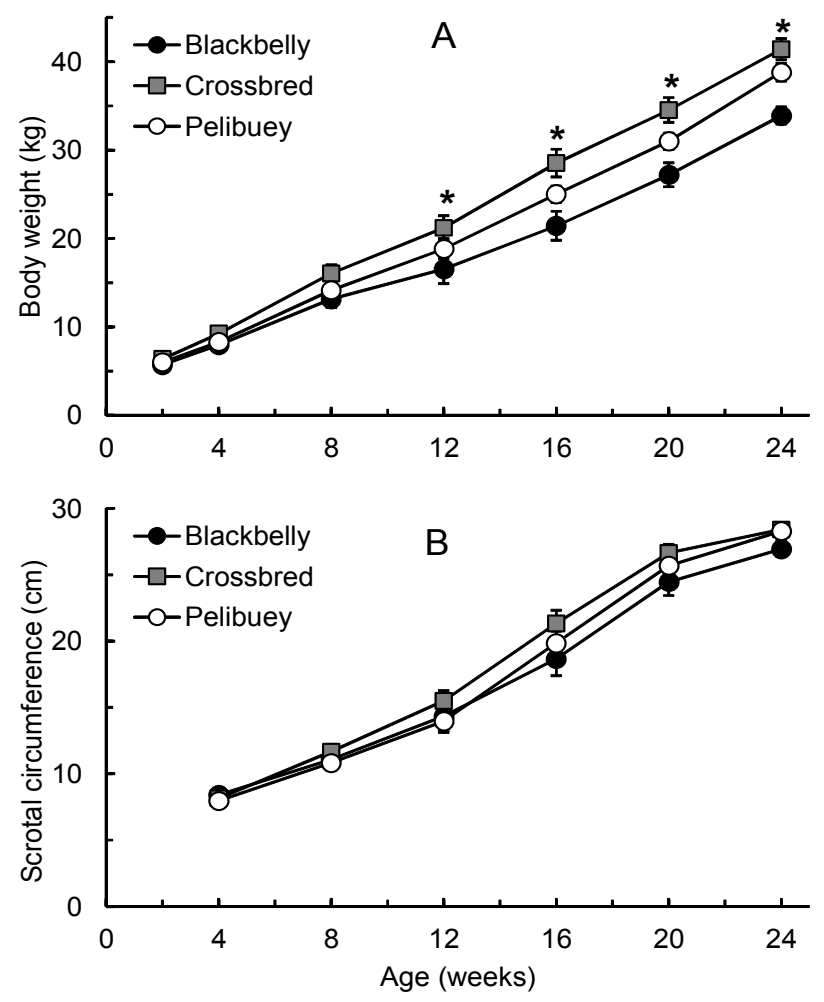

Significant differences between genotypes, within age, are denoted by * $(P<0.05)$ at wk 12 (Blackbellya, Pelibueyab, Crossbred $\left.{ }^{b}\right), 16$ and 20 (Blackbellya, Pelibuey ${ }^{b}$, Crossbred $^{\mathrm{C}}$ ), and 24 (Blackbellya, Pelibueyb, Crossbredb). Note that at some time points the symbols are larger than the SE bar. 
muy tempranas. La amplitud de los pulsos a los 28 días fue similar a la reportada previamente para razas de lana de la misma edad(22). La frecuencia de pulsos a las 2 semanas fue mayor en los corderos del presente estudio, comparado con corderos de razas de lana de un mes de edad(22), en los que la frecuencia no alcanzó valores comparables a los corderos de este estudio hasta los 90 días.

Un evento importante durante el desarrollo sexual es un aumento temprano de la concentración de LH. En este estudio, aumentos significativos de la concentración de LH y la amplitud de los pulsos se detectaron por primera vez a las 4 semanas de edad, lo cual coincide con estudios anteriores en corderos Blackbelly $(14)$ y varias razas de lana $(1,3,5,19)$. El aumento temprano y luego sostenido de la concentración de LH hasta la semana 12 resulta de una disminución en la sensibilidad del eje hipotálamo-hipófisis a la retroalimentación negativa de los esteroides, que representa tal vez el inicio del proceso puberal(14). El subsiguiente descenso de los parámetros de los perfiles de LH después de la semana 12 (Estudio 1) y de la respuesta a GnRH después de la semana 16 (Estudio 2), en conjunto con el aumento de las concentraciones de $\mathrm{T}$, pudieron ser consecuencia del establecimiento de los mecanismos de retroalimentación negativa de los esteroides gonadales(2,23).

El aumento progresivo de las concentraciones y la amplitud de los pulsos de $\mathrm{T}$ coincide con lo encontrado anteriormente en razas de lana $(1,4,22) ;$ éste es inicialmente desencadenado por el aumento de la frecuencia y (0) magnitud de los pulsos de $\mathrm{LH}(4,21,24)$, y por el incremento de la actividad esteroidogénica testicular y la capacidad de respuesta a la estimulación $\mathrm{LH}(2,21,22)$. A pesar de la disminución de la secreción de LH después de la semana 12, las concentraciones de $\mathrm{T}$ continuaron aumentando hasta la semana 24; se ha observado que durante este tiempo aumenta el número de células de Leydig(25) y el número de receptores para $\mathrm{LH}(22,26)$, se mejora la actividad enzimática genotype and genotype by age interaction were not significant. The effect of age was significant $(P<0.001)$, and SC augmented as the age increased (Figure 2B).

\section{DISCUSSION}

Previous studies have found a relationship between the endocrine changes during the prepubertal development and some reproductive characteristics in sheep. The age at which $\mathrm{LH}$ concentration starts increasing seems to be related with the precocity and prolificacy of the breed, as LH increase is observed earlier in life in more precocious(1) and more prolific breeds $(5,19)$. Therefore, the lack of differences between genotypes for any of the variables of the $\mathrm{LH}$ and $\mathrm{T}$ profiles was unexpected, as several authors have reported that Blackbelly is a more precocious and prolific breed, as compared with Pelibuey $(7,8,10,12)$. It is possible that differences between sexes in the endocrine mechanisms leading to puberty $(20)$ had hidden subtle differences between males of these breeds. Alternatively, as the breeds used in the present study have similar origin(6), they are not too different from each other, which accounted for a similar endocrine development.

Mean LH concentration followed a pattern similar to that reported previously for Blackbelly ram lambs(14) and for several wool breeds $(1,2,3,21,22)$. There were small concentrations during the first weeks of life(2,3), reaching maximum concentrations between 11 and 13 wk of age, depending on the breed $(3,22)$, followed by a decline or minimal variations thereafter. In the present study, low amplitude pulses were detected as early as $2 \mathrm{wk}$ of age, which agrees with that reported for wool breeds $(1,21)$, indicating that a functional, yet immature, reproductive axis exists at very early age. Pulse amplitude at $28 \mathrm{~d}$ of age was similar to that previously reported for wool breeds at the same age(22). Pulse frequency at 2 wk of age is higher in lambs from the present study, as compared 
y hay mayor disponibilidad de colesterol al interior de las células(26).

Al comienzo del estudio, la dosis baja de GnRH (3 $\mathrm{ng} \bullet \mathrm{kg}^{-1}$ ) fue considerada como la "dosis fisiológica", ya que previamente esta dosis había inducido aumentos de $\mathrm{LH}$ con características similares a los pulsos naturales de LH en machos adultos(17). Sin embargo, después de analizar los datos fue evidente que $\Delta \mathrm{LH}$ para la dosis media ( $9 \mathrm{ng} \mathrm{GnRH} \bullet \mathrm{kg}^{-1}$ ) fue más parecida a la amplitud de los pulsos de LH del estudio 1 que la dosis baja; por lo tanto, la dosis media podría considerarse más cercana a las descargas fisiológicas de $\mathrm{GnRH}$.

Las diferencias entre genotipos en la respuesta de $\mathrm{LH}$ a la GnRH no siempre fueron evidentes cuando se desafió con la dosis más baja; sin embargo, con las dosis altas la altura del pico y $\Delta \mathrm{LH}$ fueron mayores en los corderos Pelibuey, lo que indica una mayor capacidad de respuesta de la hipófisis en esta raza(27), principalmente en las semanas 8 y 12 . Esto coincide con los datos del estudio 1 , donde los cambios en las características del perfil de LH fueron evidentes a esas edades, los cuales parecen ser eventos endocrinos importantes asociados con el proceso de pubertad. Estos cambios endocrinos están temporalmente asociados con el establecimiento de la espermatogénesis(14) y con el desarrollo y mayor tasa de crecimiento testicular en corderos Blackbelly(15). En el presente estudio no se hicieron observaciones respecto al inicio de la pubertad; sin embargo, con base en estudios anteriores en condiciones similares(14), la espermatogénesis comienza entre las 9 y 12 semanas de edad y se completa (basado en la presencia de espermátidas alargadas en la mayoría de los túbulos seminíferos) hacia las semanas 15 a 18 , aunque no se haya alcanzado la máxima capacidad; por otra parte, bajo condiciones similares, se observó la presencia de espermatozoides en la cola del epidídimo a las 18 semanas de edad(28).

La edad en que los corderos Pelibuey alcanzaron la mayor respuesta de $\mathrm{LH}$ coincide con lo with lambs from wool breeds at one month of age(22), in which frequency did not reach values comparable to our lambs until $90 \mathrm{~d}$ of age.

An important event during sexual development is an early rise in $\mathrm{LH}$ concentration. In our study, a significant increase in mean $\mathrm{LH}$ concentration and pulse amplitude was first detected at 4 wk of age, which is in agreement with previous studies in Blackbelly(14) and several wool sheep $(1,3,5,19)$. The early and then sustained increase in $\mathrm{LH}$ concentration until wk 12 results from a decrease in the sensitivity of the hypothalamus-hypophysis axis to steroid negative feedback, representing perhaps the beginning of the pubertal process(14). The subsequent decline in the LH profile parameters after wk 12 (Study 1 ) and in the response to GnRH after wk 16 (Study 2), in conjunction with increasing $\mathrm{T}$ values, were probably consequence of the establishment of the mechanisms of negative feedback of gonadal steroids $(2,23)$.

The progressive increase of $\mathrm{T}$ concentration and pulse amplitude is in agreement with previous reports for wool breeds $(1,4,22)$; it is initially triggered by the increased frequency and (or) magnitude of $\mathrm{LH}$ pulses $(4,21,24)$, and the enhanced testicular steroidogenic activity and responsiveness to $\mathrm{LH}$ stimulation(2,21,22). Albeit the decreased $\mathrm{LH}$ secretion after wk 12, T concentrations continued rising until wk 24; in addition to the greater number of Leydig cells $(25)$, there are also increased numbers of LH receptors $(22,26)$, enhanced enzymatic activity, and more availability of cholesterol to the interior of the cells(26).

At the beginning of the study, the small dose of $\mathrm{GnRH}$ (3 $\mathrm{ng} \bullet \mathrm{kg}^{-1}$ ) was considered the "physiological dose", as previously it had induced $\mathrm{LH}$ rises with characteristics similar to the natural LH pulses in adult rams(17). However, after analyzing the data, it was evident that $\Delta \mathrm{LH}$ for

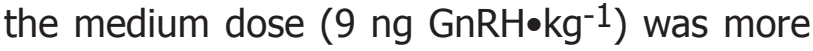
comparable to the LH pulse amplitude from the 
observado en corderos Romney; en tal raza, la máxima respuesta a GnRH se observó a las 6 semanas de edad, y la magnitud de la respuesta disminuyó a medida que la edad avanzaba(29). La interacción dosis $x$ genotipos sobre la respuesta de LH encontrada en el presente estudio, contrasta con información previa en corderos de varias razas de lana (Finn, Finngalway y High Fertility); en esta última, a los corderos se les administró entre 5 y $100 \mu \mathrm{g}$ de $\mathrm{GnRH}$, a las 4 y 8 semanas de edad, y la interacción raza por dosis no fue significativa a ninguna edad(30). Una posible explicación para esta discrepancia es que las dosis utilizadas en corderos de lana fueron tan altas que la respuesta provocada fue máxima a cualquier dosis; en contraste, la dosis máxima que se administró a los corderos del presente trabajo fue alrededor de $1 \mu \mathrm{g}$ al final del estudio, lo cual pudo permitir cierto grado de discriminación en la magnitud del estímulo con GnRH.

Los hallazgos en la secreción de T después de la administración de GnRH apoyan los resultados del primer estudio, en el cual las interacciones de genotipo por dosis y el genotipo por edad no fueron significativas para ninguna de las variables evaluadas. A pesar de que existieron diferencias entre genotipos en la respuesta de $\mathrm{LH}$ a la $\mathrm{GnRH}$, éstas no fueron suficientes para inducir claras diferencias entre genotipos en la respuesta testicular, en términos de secreción de T. El efecto de la dosis de GnRH sobre la secreción de LH fue significativo y la respuesta fue siempre de una manera dosis dependiente, lo que concuerda con estudios similares anteriores $(30,31)$. La respuesta de la testosterona también fue dosis dependiente, lo que sugiere que en los corderos en desarrollo, los testículos son capaces de detectar las diferentes concentraciones de $\mathrm{LH}$ inducidas por $\mathrm{GnRH}$, y liberar $\mathrm{T}$ consecuentemente. Estos hallazgos contrastan con los reportados previamente para machos adultos, en los cuales, a pesar de la diferente respuesta de $\mathrm{LH}$ a dosis crecientes de $\mathrm{GnRH}$, la magnitud de la respuesta de $\mathrm{T}$ fue la misma, independientemente de la dosis de $\mathrm{GnRH}(32)$. Por otro lado, en un estudio con study 1 than the small dose was; therefore, the medium dose might be considered closer to physiological $\mathrm{GnRH}$ discharges.

Differences among genotypes in the $\mathrm{LH}$ response to $\mathrm{GnRH}$ were not always evident when challenged with the small dose; however, with the larger doses peak height and $\Delta \mathrm{LH}$ were greater in Pelibuey lambs, which indicates a greater capacity of response of the hypophysis(27) in this breed, primarily on wk 8 and 12 . This is in agreement with data from Study 1, where changes in the characteristics of $\mathrm{LH}$ profile are evident at those ages, which seem to be important endocrine events associated to the process of puberty. These endocrine changes are temporally associated with the establishment of spermatogenesis(14), testis development and the greatest rate of testicular growth(15) in Blackbelly ram lambs. In the present study no observations were made regarding the puberty status; however, based on previous studies under similar conditions(14), spermatogenesis starts between 9 and 12 wk of age and is completed (based on the presence of elongated spermatids in most of the seminiferous tubules) by wk 15 to 18 , albeit not reached full capacity; furthermore, under similar conditions, sperms were present in the cauda epididymidis at 18 wk of age(28).

The age at which Pelibuey lambs reached the greatest $\mathrm{LH}$ response to $\mathrm{GnRH}$ is in agreement with a previous report for Romney lambs; in such breed, the maximal response to $\mathrm{GnRH}$ was observed at $6 \mathrm{wk}$ of age, and the magnitude of the response decreased as the lambs became older(29). The dose by genotype interaction on $\mathrm{LH}$ response to $\mathrm{GnRH}$ found in the present study is in contrast with a study in lambs of several wool breeds (Finn, Finngalway and High Fertility). In the last, lambs were administered between 5 and $100 \mu \mathrm{g} \mathrm{GnRH}$ at 4 and $8 \mathrm{wk}$ of age, and the breed by dose interaction was no significant at any age(30). A possible explanation to this disagreement is that the doses used with the wool lambs were so high that the response elicited was maximal at any dose; in 
toros, el AUC de respuesta de T fue acorde con la dosis de $\mathrm{GnRH}(33)$; la respuesta bifásica de $\mathrm{LH}$, provocada al administrar dosis altas de $\mathrm{GnRH}$, prolonga la estimulación testicular y, por lo tanto, favorece la secreción de mayores cantidades de $\mathrm{T}(33,34)$.

A pesar de que los corderos Pelibuey mostraron una mayor capacidad de respuesta a la $\mathrm{GnRH}$, esta capacidad aumentada no se hizo evidente ni tuvo repercusión en el proceso fisiológico normal de maduración endocrina (estudio 1). Es posible que en condiciones fisiológicas, el hipotálamo genere sólo los estímulos necesarios para desencadenar los cambios endocrinos asociados con el inicio de la pubertad y el desarrollo sexual, por lo cual, los perfiles de LH y $\mathrm{T}$ no difirieron entre genotipos(27). La respuesta diferenciada a la administración de $\mathrm{GnRH}$ entre genotipos o entre individuos, sin embargo, podría ser de algún valor para predecir el comportamiento reproductivo del animal en la vida adulta o de su progenie(27,35), pero tal posibilidad no puede ser verificada a partir de los datos del presente estudio.

No se detectaron diferencias en el desarrollo de la CE, a pesar del mayor peso de los corderos Pelibuey y de los cruzados a partir de la semana 12; esto no concuerda con estudios anteriores, donde las diferencias en la CE durante el desarrollo de los corderos se explicaron basándose en los diferentes pesos de las razas(36). El pequeño número de corderos por grupo pudo haber influido para no detectar diferencias significativas entre los genotipos.

\section{CONCLUSIONES E IMPLICACIONES}

En conclusión, el desarrollo endocrino sexual de los corderos Blackbelly, Pelibuey y sus cruzas no difiere cuando los perfiles de LH y T circulantes son evaluados; sin embargo, los corderos Pelibuey muestran una mayor capacidad de respuesta de la LH, cuando son desafiados con dosis fisiológicas y supra fisiológicas de $\mathrm{GnRH}$. Esta respuesta diferenciada se observa durante un período en la vida del contrast, the maximum dose administered to our lambs was close to $1 \mu \mathrm{g}$ at the end of the study, which could have allowed for some degree of discrimination in the magnitude of the $\mathrm{GnRH}$ stimulus.

Findings in T secretion after GnRH administration support the results of the first study, in which the interactions of genotype by dose and genotype by age were not significant for any of the variables evaluated. Even though the differences between genotypes in the $\mathrm{LH}$ response to $\mathrm{GnRH}$, they did not suffice to induce clear differences between genotypes in the testicular response, in terms of $\mathrm{T}$ secretion. The effect of $\mathrm{GnRH}$ dose on $\mathrm{LH}$ secretion was significant and the response was always in a dose-dependent manner, which agrees with previous similar studies $(30,31)$. Testosterone response was also dose-dependent, which suggests that in developing lambs the testes are able to detect the different LH concentrations induced by $\mathrm{GnRH}$, and release $\mathrm{T}$ accordingly. These findings contrast with those previously reported for adult rams; despite the differential $\mathrm{LH}$ response to graded doses of $\mathrm{GnRH}$, the magnitude of $\mathrm{T}$ response was the same regardless the $\mathrm{GnRH}$ dose(32). On the other hand, in a study with bulls, AUC of T response was related to $\mathrm{GnRH}$ dose(33). The biphasic $\mathrm{LH}$ response, elicited when high doses of GnRH are administered, accounted for extended testis stimulation, and therefore, greater amounts of $T$ were secreted $(33,34)$.

Even though Pelibuey lambs showed a greater capacity of response to $\mathrm{GnRH}$, such enhanced capacity did not become evident nor had repercussion on the normal physiological process of endocrine maturation (Study 1). It is possible that under physiological conditions the hypothalamus generates only the necessary stimulus to trigger the endocrine changes associated with the onset of puberty and sexual development, and, therefore, the variables of the non-stimulated $\mathrm{LH}$ and $\mathrm{T}$ profiles did not differ between genotypes(27). The differential response to $\mathrm{GnRH}$ between genotypes or 
animal, en el que se producen importantes cambios en el desarrollo de la función testicular. Se requiere investigación adicional para determinar si esas diferencias tienen implicaciones sobre la capacidad reproductiva de los machos adultos.

\section{AGRADECIMIENTOS}

Se agradece a Leticia García y Patricia Sánchez por la asistencia técnica en el laboratorio, a Cristina Huerta y Martín Martínez por ayudar en la colección de las muestras y el cuidado de los corderos, a Anahy Vargas por la traducción al español. LJ Montiel-Olguín fue estudiante de posgrado, cuyo programa académico fue apoyado por el CONACYT, bajo la supervisión del último autor (HJS).

\section{LITERATURA CITADA}

1. Echternkamp SE, Lunstra DD. Relationship between LH and testicular development in progesterone-implanted prepubertal ram lambs. J Anim Sci 1984;59(2):441-453.

2. Rawlings NC, Churchill IJ, Currie WD, Joseph IBJK. Maturational changes in opioidergic control of luteinizing hormone and follicle-stimulating hormone in ram lambs. J Reprod Fertil 1991;93:1-7.

3. Lee VW, Cumming IA, de Kretser DM, Findlay JK, Hudson $B$, Keogh EJ. Regulation of gonadotrophin secretion in rams from birth to sexual maturity. I. Plasma LH, FSH and testosterone levels. J Reprod Fertil 1976;46:1-6.

4. Klindt J, Ohlson DL, Davis SL, Schanbacher BD. Ontogeny of growth hormone, prolactin, luteinizing hormone, and testosterone secretory patterns in the ram. Biol Reprod 1985;33(2):436-444.

5. Glatzel P. Variation in hormonal feedback and reproductive performance in rams of different genotypes from birth through puberty. Theriogenology 1988;30(4):763-776.

6. Mason IL. Prolific tropical sheep 1980; Paper 17, FAO Rome.

7. González-Reyna A, Valencia MJ, Foote WC, Murphy BD. Hair sheep in México: reproduction in the Pelibuey sheep. Anim Breed Abstr 1991;59:509-524.

8. Segura JC, Sarmiento L, Rojas O. Productivity of Pelibuey and Blackbelly ewes in Mexico under extensive management. Small Ruminant Res 1996;21(1);57-62.

9. Young LD, Fahmy MH, Torres HG. The use of prolific sheep: North America. In: MH Fahmy editor. Prolific Sheep. CAB International, Wallingford United Kingdom; 1996:289-349. between individuals, however, might be of some value to predict the reproductive performance of the ram in the adult life or its progeny $(27,35)$, but such possibility cannot be verified from data of the present study.

No differences were detected in SC development, albeit the greater BW in Pelibuey and crossbred lambs from wk 12, which disagree with previous studies, where differences in SC in developing ram lambs were explained based on differences in breed BW(36). The small number of lambs per group might have accounted for not detecting significant differences among genotypes.

\section{CONCLUSIONS AND IMPLICATIONS}

In conclusion, the sexual endocrine development of Blackbelly, Pelibuey and their crosses does not differ when the non-stimulated circulating profiles of $\mathrm{LH}$ and $\mathrm{T}$ are evaluated; however, Pelibuey lambs showed a greater capacity of LH response, when challenged with physiological and supra physiological doses of $\mathrm{GnRH}$. This differential response was observed during a period in the life, when important changes occur in the development of the testicular function of the rams. Whether or not such differences have implications on the reproductive capacity of the adult rams deserves additional research.

\section{ACKNOWLEDGEMENTS}

Thanks to Leticia García and Patricia Sánchez for technical assistance in the laboratory, Cristina Huerta and Martín Martínez for helping in collecting samples and caring for the lambs. LJ Montiel-Olguín is a former graduate student, whose academic program was supported by CONACYT, under the supervision of the last author (HJS).

End of english version 
10. Valencia-Zarazúa M, González-Padilla E. Pelibuey sheep in Mexico. In: Fitzhugh HA, Bradford GE editors. Hair sheep of Western Africa and the Americas. A genetic resource for the tropics 1st ed. Boulder CO: Westview Press; 1983:5573.

11. Heredia AM, Velázquez MPA, Zarco QLA. Efecto de genotipo sobre el peso y edad a la pubertad en corderas Pelibuey, Blackbelly y sus cruzas [resumen]. Reunión Nacional de Investigación Pecuaria. Cuernavaca, Mor. México. 1996;322.

12. Wildeus S. Hair sheep genetic resources and their contribution to diversified small ruminant production in the United States. J Anim Sci 1997;75:630-640.

13. Zavala-Elizarraraz R, Ortiz-Ortiz JR, Ramón-Ugalde JP, Montalvo-Morales P, Sierra-Vásquez A, Sanginés-García JR. Pubertad en hembras de cinco razas ovinas de pelo en condiciones de trópico seco. Zoot Trop 2008;26(4):465473.

14. Herrera-Alarcón J, Villagómez-Amezcua E, González-Padilla E, Jiménez-Severiano H. Stereological study of postnatal testicular development in Blackbelly sheep. Theriogenology 2007;68(4):582-591.

15. Jiménez-Severiano $H$, Reynoso ML, Roman-Ponce SI, Robledo VM. Evaluation of mathematical models to describe testicular growth in Blackbelly ram lambs. Theriogenology 2010;74(7):1107-1114.

16. Merriam GR, Wachter KW. Algorithms for the study of episodic hormone secretion. American J Physiol 1982;243(4):E310-E318.

17. Jiménez-Severiano $\mathrm{H}$. Endocrine and testicular responses of male cattle and sheep to chronic treatment with analogs of GnRH [unpublished PhD dissertation]. University of Nebraska; Lincoln 2001.

18. Jiménez-Severiano $H$, Jiménez-Krassel $F$, Menéndez-Trejo $M$. Endocrine and testicular responses of prepubertal Zebu crossbred bulls to active immunization against testosterone. Anim Reprod Sci 1996;41(3-4):169-81.

19. Lunstra DD, Echternkamp SE. Repetitive testicular biopsy in the ram during pubertal development. Theriogenology 1988;29(4):803-810.

20. Foster DL, Jackson LM. Puberty in sheep. In: Neill JD editor. Knobil and Neill's Physiology of Reproduction. Third ed vol.1. San Diego CA; Elsevier Academic Press; 2006:2127-2176.

21. Foster DL, Mickelson IH, Ryan KD, Coon GA, Drongowski RA, Holt JA. Ontogeny of pulsatile luteinizing hormone and testosterone secretion in male lambs. Endocrinology 1977;102(4):1137-1146.

22. Yarney TA, Sanford LM. Pubertal changes in the secretion of gonadotropic hormones, testicular gonadotropic receptors and testicular function in the ram. Domest Anim Endocrinol 1989;6(3):219-229.

23. Schanbacher BD. Testosterone regulation of luteinizing hormone and follicle stimulating hormone secretion in young lambs. J Anim Sci 1980;51(3):679-684.
24. Chase DJ, Schanbacher BD, Lunstra DD. Effects of pulsatile and continuous luteinizing hormone (LH) infusions on testosterone responses to $\mathrm{LH}$ in rams actively immunized against gonadotropin-releasing hormone. Endocrinology 1988;123(2):816-826.

25. Monet-Kuntz C, Hochereau-de Reviers MT, Terqui M. Variations in testicular androgen receptors and histology of the lamb testis from birth to puberty. J Reprod Fertil 1984;70:203-210.

26. O'Shaughnessy PJ, Willerton L, Baker PJ. Changes in Leydig cell gene expression during development in the mouse. Biol Reprod 2002;66(4):966-975.

27. Evans NP, McNeilly JR, Springbett AJ, Webb R. Alterations in pituitary gland sensitivity in ram lambs to physiological doses of gonadotrophin-releasing hormone (GnRH), after divergent selection based on the luteinizing hormone response to a pharmacological GnRH challenge. J Reprod Fertil 1991;93:559-567.

28. Vargas-Velázquez AD, Jiménez-Severiano H. Estudio morfométrico de los epidídimos durante el desarrollo postnatal de corderos Barbados Blackbelly. Rev Mex Cienc Pecu 2016;7(1):53-68.

29. Wilson PR, Lapwood KR. Studies of reproductive development in Romney rams. I. Basal levels and plasma profiles of LH, testosterone and prolactin. Biol Reprod 1979;20(4):965970.

30. Hanrahan JP, Quirke JF, Gosling JP. Effect of lamb age, breed and sex on plasma $\mathrm{LH}$ after administration of $\mathrm{GnRH}$. J Anim Sci 1981;61:281-288.

31. Tyrrell RN, Starr BG, Restall BJ, Donnelly JB. Repeatability of $\mathrm{LH}$ responses by lambs to monthly challenge with synthetic gonadotrophin releasing hormone (GnRH). Anim Reprod Sci 1980;3(2):155-160.

32. Jiménez-Severiano $H, D^{\prime} O c c h i o ~ M J$, Lunstra DD, Mussard $M L$, Davis TL, Enright WJ, Kinder JE. Comparative response of rams and bulls to long-term treatment with gonadotropinreleasing hormone analogs. Anim Reprod Sci 2007;98(34):204-224.

33. Post TB, Reich MM, Bindon BM. Characterization of LH and testosterone responses to intramuscular injection of $\mathrm{GnRH}$ in tropical post pubertal bulls. Theriogenology 1987;27(2):305-315.

34. Evans NP, McNeilly JR, Webb R. Effects of indirect selection for pituitary responsiveness to gonadotropin-releasing hormone on the storage and release of luteinizing hormone and follicle-stimulating hormone in prepubertal male lambs. Biol Reprod 1995;53(2):237-243.

35. Haley CS, Lee GJ, Fordyce M, Baxter G, Land RB, Webb R. Study of LH response to $\mathrm{GnRH}$ in the young male as a criterion of genetic merit for female reproduction in sheep. J Reprod Fert 1989;86:119-133.

36. Notter DR, Lucas JR, McClaugherty FS, Copenhaver JS. Breed group differences in testicular growth patterns in springborn ram lambs. J Anim Sci 1985;60(3):622-631. 